\title{
FROM BROWNIAN DYNAMICS TO MARKOV CHAIN: AN ION CHANNEL EXAMPLE
}

\author{
WAN CHEN ${ }^{1}, \quad$ RADEK ERBAN ${ }^{1,2}$, AND S. JONATHAN CHAPMAN ${ }^{1,3}$
}

\begin{abstract}
A discrete rate theory for general multi-ion channels is presented, in which the continuous dynamics of ion diffusion is reduced to transitions between Markovian discrete states. In an open channel, the ion permeation process involves three types of events: an ion entering the channel, an ion escaping from the channel, or an ion hopping between different energy minima in the channel. The continuous dynamics leads to a hierarchy of Fokker-Planck equations, indexed by channel occupancy. From these the mean escape times and splitting probabilities (denoting from which side an ion has escaped) can be calculated. By equating these with the corresponding expressions from the Markov model the Markovian transition rates can be determined. The theory is illustrated with a two-ion one-well channel. The stationary probability of states is compared with that from both Brownian dynamics simulation and the hierarchical Fokker-Planck equations. The conductivity of the channel is also studied, and the optimal geometry maximizing ion flux is computed.
\end{abstract}

Key words. ion hopping, hierarchical Fokker-Planck equations, transition rates, optimal flux

1. Introduction. The membrane of a eukaryotic cell is mainly composed of a lipid bilayer, which is impermeable to water solvated ions [2]. Ion channels are nanopores formed by transmembrane proteins; they allow ions to flow through and act as biological valves connecting the intracellular with extracellular domains. Ion channels are the main mechanism by which cells control the intracellular concentration of chemical species, as well as the potential gradient across the membrane. As such they play important roles in maintaining various functions of plant, animal and human cells.

There are two main features which distinguish ion channels from other nanoscale porous media. Firstly, they may be selective, distinguishing between the charge and size of ions; for example, the potassium $\mathrm{K}^{+}$channel conducts potassium ions at a rate $10^{4}$ times faster than it does sodium ions [10]. Secondly, their conformations may change between open and closed states in response to an external stimulus such as a voltage gradient, ligand binding, or $\mathrm{pH}$ value.

The molecular structure of many ion channels has been revealed by X-ray crystallography in recent decades, which provides insight into their features and function. For example, the potassium $\mathrm{K}^{+}$channel is composed of four identical subunits which create a cavity connecting the cell interior to a selectivity filter at the outer end of the pore [10]. The narrow selectivity filter is only $12 \AA$ long and about $3 \AA$ wide, which forces potassium ions with Pauling radius $1.33 \AA$ to shed their hydrating waters to enter and pass in a single-file fashion. The oxygen atoms of four carbonyl groups form four rings around the selectivity filter, which generate local minima called binding sites in the overall energy landscape to coordinate the dehydrated ions.

Mathematical models for ion channels include molecular dynamics (MD), Brownian dynamics (BD) and continuum theory (Poisson-Nernst-Planck equations) in descending order of resolution [8, 7, 15]. Molecular dynamics provides the most detailed description by mimicking the motions and interactions of all atoms (from membrane proteins to free ions and even individual water molecules) at the molecular level [3, 18, 14]. Since the relaxation of water molecules happens at the fastest timescale of $1 \mathrm{fs}$, the time step of an MD simulation has to be very small, and one needs to evolve a system of thousands of particles up to of the order of $0.1 \mathrm{~ms}$ to observe ion conduction. Such a simulation is obviously computationally

\footnotetext{
${ }^{1}$ Mathematical Institute, University of Oxford, 24-29 St Giles', Oxford OX1 3LB, United Kingdom

${ }^{2}$ E-mail: erban@maths.ox.ac.uk

${ }^{3}$ E-mail: chapman@maths.ox.ac.uk
} 
intensive, but much shorter simulations (of the order of $10 \mathrm{ps}$ ) can be used to obtain information about the local potential energy and the effective diffusion coefficient of ions, which can then be fed into BD simulations.

Brownian dynamics [22, 9, 16, 6] is a more coarse-grained simulation which replaces the solvent molecules (water) as a continuum, and represent their influence by a dialectric constant and stochastic forcing. The fluctuations of membrane proteins are ignored and the channel is approximated by a solid boundary. Because the dynamics of water and proteins are no longer included, a relatively long time step can be used, which greatly reduces the computational cost. In this paper, we focus on this level of resolution, and introduce a discrete rate theory that is based on observations from BD.

The continuum model [5, 21, 17] calculates the potential energy by a mean-field approximation of average ion positions, which yields a Poisson equation, and then formulates a Boltzmann equation (in equilibrium) or a Nernst-Planck equation (in non-equilibrium) for the ion concentration. These continuum partial differential equations (PDEs) can be solved efficiently; however the individual ion-ion interaction is missing in this mean-field assumption which then fails to predict some properties (e.g. saturation). Comparisons of BD and continuum theories in different channel configurations are presented in [9, 16].

Recently several hybrid models combining MD and the theory of stochastic processes have been proposed, which are able to include molecular details and access long time scales while keeping computational cost low. One idea is to apply the Eyring rate theory to the ion permeation process using the potential of mean force (PMF) calculated using MD. This is based on the assumption that channels have some binding sites, and ions pass through by a hopping mechanism: an ion fluctuates around a certain site before it obtains enough energy to overcome the energy barrier and hops into the adjacent vacant site. This ion hopping mechanism has been revealed by MD in channels with binding sites [3, 4, 14]. In addition, the single file diffusion constraint imposed by the narrowness of the channel assures that ions cannot cross each other in the channel. Therefore, the continuous dynamics of ion diffusion can be represented by transitions between discrete Markovian states.

The Eyring rate theory was originally designed for chemical reactions in the 1930s, with transition rates proportional to the exponential of the energy barrier and distance between binding sites [11] (as shown in [8] this overestimates the physical barrier in the ion-crossing process). A novel theory was proposed recently in [1] for a one-dimensional channel with sawtooth-like PMF, in which the transition rates are not approximated using the energy barrier but are obtained as the product of total escape rate from one binding site and the splitting probability determining the relative chance of landing in each neighbouring site. [1] showed that an optimal size of binding site maximizes the ionic flux if the applied voltage exceeds a threshold. They assume the channel is occupied by at most one ion, whereby the resulting system forms a single Markov chain, and the rates can be solved explicitly. In the multi-ion channel considered here, ion-ion interactions as well as the higher dimension of the energy landscape mean that the complexity of the rate theory is greatly increased.

In this paper, we present a general discrete rate theory for a multi-ion channel, and compare it with $\mathrm{BD}$. The ion permeation process involves ion hopping, ion escaping and ion entering. For the purposes of this work we assume ion entry rates are known and focus on calculating the other rates in terms of the mean escape time and splitting probability. Because of the complicated network between states the rates are more intricately related to these quantities than in the single ion case. Moreover, since analytical solutions for the mean escape time and splitting probability are not available, these must be determined by solving the corresponding PDEs numerically. The theory is illustrated by a two-ion channel with one binding site and two ion sources. We show that, as with the one-ion channel, there exists an 


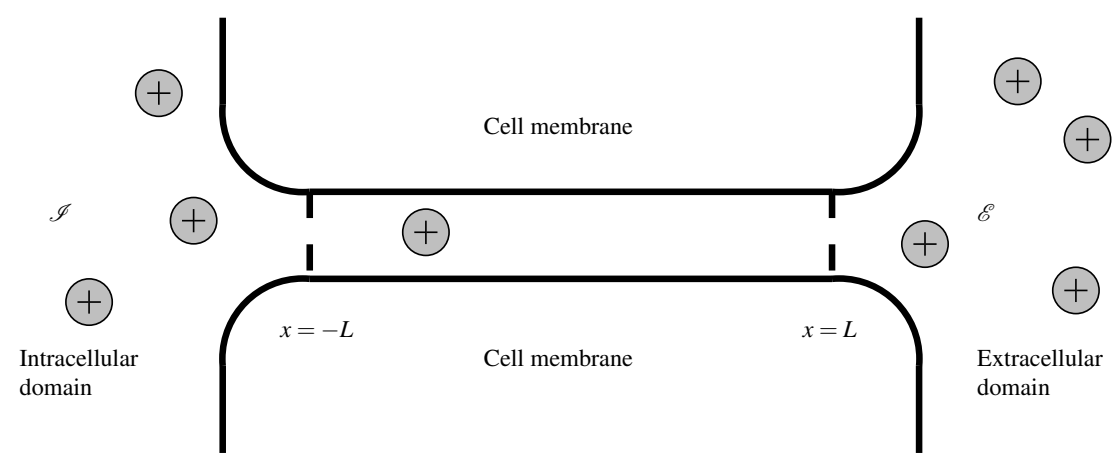

FIG. 2.1. A schematic structure of a channel. $x=-L$ and $x=L$ are the (artificial) left and right boundaries connecting large reservoirs of electrolyte in and outside the cell respectively. $\mathscr{I}$ and $\mathscr{E}$ represent the overall intracellular and extracellular environments, respectively.

optimal shape for the external potential that allows a maximal flux.

The structure of this paper is as follows. In Section 2, we introduce a general theory for a multi-ion channel with a maximal capacity of $N$ ions. We first present BD simulations and formulate an equivalent cascade of hierarchical Fokker-Planck equations for the probability distribution of ions. An illustrative example of a 2-ion channel is discussed and the probability distribution from the histogram of $\mathrm{BD}$ and the solution of the Fokker-Planck equation are compared. Next, a discrete rate theory framework is presented in Section 3 and the transition rates calculated. The 2-ion channel is revisited in this framework, and the result is compared with that from BD. In Section 4, we apply the theory to study the dependence of channel conduction on different parameters such as the diffusion coefficient, ion entry rate and depth of potential wells. In particular, we study the effect of the geometry of the external potential in Section 5. We conclude by discussing the advantages and limitations of this method and possible applications and extensions in Section 6 .

2. Brownian dynamics. In this section, we present the theoretical framework of BD simulation. Since we are interested in studying the ion permeation process, which occurs on a time scale of $10^{-7} \mathrm{~s}$, and since conformational changes occur on a timescale of $10^{-3} \mathrm{~s}$, we assume that channel is always open and does not change its conformation.

Since the channel is very narrow and the ions pass through in single file [14], we will suppose that the motion is one-dimensional, that is, the centres of the ions will be constrained to lie along a line. The generalisation to a fully three dimensional channel is algebraically complicated but conceptually straightforward. Since ions cannot pass each other in one dimension, we may neglect the finite size of the ions and model them as point particles with charge.

We define the maximal capacity of a channel to be $N$, so that it can hold up to $N$ ions at one time. We denote the number of binding sites in the channel by $M$. The parameters $N$ and $M$ vary among different channels; for example, a germicidal A channel has two binding sites $(M=2)$ and single-ion occupation dominates (so that $N=1$, or perhaps $N=2$ to allow for a knock-on effect) [1, 19].

At the ends of the channel the pore opens out into the intracellular and extracellular space. A full model would include (probably continuum) models of these spaces, which would then be joined (preferably matched in terms of matched asymptotic expansions, but more likely patched) to the channel model. For our present purposes we need to introduce (artificial) interfaces (i.e. points) at the left and right ends of the channel such that an ion 
passing through these interfaces is taken to have left the channel and passed into the external domains. Without loss of generality, we suppose that the left interface connecting the channel to the intracellular domain lies at $x=-L$, and the right interface connecting the channel to the extracellular domain lies at $x=L$, as shown in Fig. 2.1. Thus an absorbing boundary condition is imposed at $x=-L$ and $x=L$.

In BD simulation of an ion channel the contribution of water molecules to the motion of a solute ion can be approximated by random collisions and an average frictional force in the evolution equation of the solute ion [22, 16]. The motion of a system of $k$ ions is given by the Langevin equation

$$
m_{i} d v_{i}=-\gamma v_{i} \mathrm{~d} t+f_{i}^{k}\left(x_{1}, \ldots, x_{k}\right) \mathrm{d} t+\gamma \sqrt{2 D} d W_{i}, \quad i=1, \ldots, k,
$$

where $x_{i}(t)$ and $v_{i}(t)$ are the location and velocity of the $i^{\text {th }}$ ion respectively. There are three forces on the right hand side of 2.1). The first term corresponds to the frictional force exerted on the ion by averaging the effect of water molecules; $\gamma$ is the frictional drag coefficient, which depends on the surrounding fluid environment. Here we assume it to be uniform so that $\gamma$ is constant. The third term is the stochastic force generated by the random collisions of water molecules; $W_{i}$ is a Wiener process and $D=k_{B} T / \gamma$ is the diffusion coefficient, where $k_{B}$ is the Boltzmann constant and $T$ is the temperature. The second term $f_{i}^{k}\left(x_{1}, \ldots, x_{k}\right)$ is the overall electric force on the $i^{\text {th }}$ ion, including interactions with all other $k-1$ ions in the channel, fixed charges in the protein, and external field across the membrane. It depends on the locations of all ions, and can be obtained (along with the diffusion coefficient) from MD simulation.

Note that a typical value of the diffusion coefficient in aqueous solutions at room temperature is $D \sim 10^{-3} \mathrm{~mm}^{2} \mathrm{~s}^{-1}$, so that the ratio $m_{i} / \gamma \sim 10^{-14} \mathrm{~s}^{-1}$. Since we usually take a time step $\Delta t>10^{-12} \mathrm{~s}$ in the simulation, the system is in an overdamped limit [8]. We may thus approximate 2.1 by the overdamped Langevin equation

$$
d x_{i}=\frac{D}{k_{B} T} f_{i}^{k}\left(x_{1}, \ldots, x_{k}\right) \mathrm{d} t+\sqrt{2 D} d W_{i}, \quad i=1, \ldots, k .
$$

The boundary conditions on 2.2 may be described as

1. When the number of ions in the channel $k$ is less than its capacity $N$, new ions are generated at the left (respectively right) end at a rate $H_{k}$ (respectively $G_{k}$ ). In principle $H_{k}$ and $G_{k}$ depend on the current locations of the $k$ ions in the channel $x_{1}, \ldots, x_{k}$ as well as the intracellular and extracellular environments $\mathscr{I}$ and $\mathscr{E}$.

Since we are in the overdamped limit we cannot simply place the incoming ions at the ends of the channel: under Brownian motion they would immediately cross the boundary and leave the channel again. Instead we place them at a position within the channel given by the positional distribution function $h\left(x ; x_{1}, \ldots, x_{k}\right)$ (or respectively $\left.g\left(x ; x_{1}, \ldots, x_{k}\right)\right)$. Note that $h$ and $g$ also depend on the positions of the existing ions. This is necessary since, for example, an ion entering the channel from the left must lie to the left of $x_{1}$, while an ion entering from the right must lie to the right of $x_{k}$. Thus, at the very least, $h$ depends on $x_{1}$ while $g$ depends on $x_{k}$.

The functions $h$ and $g$ should be chosen to make the join with the outer model as smooth as possible, as in [12, 13]. Here we simply assume that $h$ and $g$, and the rates $H_{k}$ and $G_{k}$, are given.

2. When $x_{i}(t)<-L$ or $x_{i}(t)>L$ the $i^{\text {th }}$ ion is removed from the channel.

3 . If $x_{i}(t)>x_{i+1}(t)$ for some $i$ then $x_{i}$ and $x_{i+1}$ are switched. This enforces the singlefile nature of the channel by preventing an ion overtaking its neighbour. This condition is unlikely to occur with ions in a channel due to the strong Coulomb repulsion, but may be necessary if we are interested in neutral molecules. 


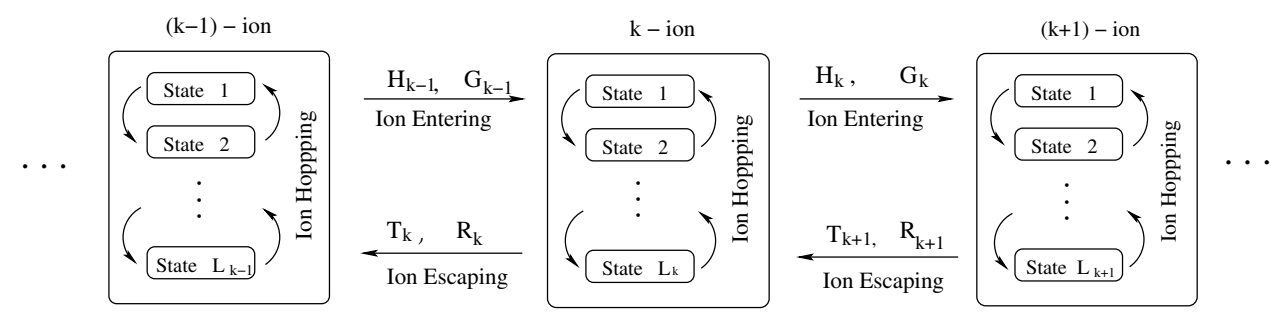

FIG. 2.2. Hierarchical Fokker-Planck Equations describe the conservation of ions in the channel. For k-ion occupancy, the transitions to and from $(k-1)$-ion and $(k+1)$-ion occupancy by ions entering and escaping are demonstrated, along with internal transitions between states.

2.1. Hierarchical Fokker-Planck equations. We denote by $P_{k}\left(x_{1}, \ldots, x_{k}, t\right)$ the probability density function for the event that there are $k$ ions in the channel at positions $x_{1}, \ldots, x_{k}$ at time $t$. Since the number of ions in the channel may run from zero to the channel capacity $N$, we have $N+1$ such probability density functions. The probability of no ion in the channel (i.e. $k=0$ ) is denoted by $P_{0}(t)$, and is independent of the spatial variable. We label the ions by the order of their locations, such that $x_{i}<x_{j}$ for $i<j$. Then the stochastic process (2.2) is equivalent to the following hierarchical system of Fokker-Planck equations:

$$
\begin{aligned}
\partial_{t} P_{k}\left(x_{1}, \ldots, x_{k}, t\right)= & D \nabla \cdot\left(\nabla P_{k}\left(x_{1}, \ldots, x_{k}, t\right)-P_{k}\left(x_{1}, \ldots, x_{k}, t\right) \frac{1}{k_{B} T} \mathbf{F}_{k}\left(x_{1}, \ldots, x_{k}, t\right)\right) \\
& -\left(H_{k}\left(x_{1}, \ldots, x_{k}\right)+G_{k}\left(x_{1}, \ldots, x_{k}\right)\right) P_{k}\left(x_{1}, \ldots, x_{k}, t\right) \\
& +H_{k-1}\left(x_{2}, \ldots, x_{k}\right) h\left(x_{1} ; x_{2}, \ldots, x_{k}\right) P_{k-1}\left(x_{2}, \ldots, x_{k}, t\right) \\
& +G_{k-1}\left(x_{1}, \ldots, x_{k-1}\right) g\left(x_{k} ; x_{1}, \ldots, x_{k-1}\right) P_{k-1}\left(x_{1}, \ldots, x_{k-1}, t\right) \\
& +T_{k+1}\left(x_{1}, \ldots, x_{k}\right)+R_{k+1}\left(x_{1}, \ldots, x_{k}\right)
\end{aligned}
$$

where $\nabla=\left(\partial_{x_{1}}, \ldots, \partial_{x_{k}}\right), \mathbf{F}_{k}=\left(f_{1}^{k}, \ldots, f_{k}^{k}\right) \in \mathbb{R}^{k}$, and

$$
\begin{gathered}
T_{k+1}\left(x_{2}, \ldots, x_{k+1}\right)=D\left(\frac{\partial P_{k+1}}{\partial x_{1}}-P_{k+1} \frac{1}{k_{B} T} f_{1}^{k+1}\right)\left(-L, x_{2}, \ldots, x_{k+1}\right), \\
R_{k+1}\left(x_{1}, \ldots, x_{k}\right)=-D\left(\frac{\partial P_{k+1}}{\partial x_{k+1}}-P_{k+1} \frac{1}{k_{B} T} f_{k+1}^{k+1}\right)\left(x_{1}, \ldots, x_{k}, L\right),
\end{gathered}
$$

where $k=0, \ldots, N$ and we use the convention that $P_{-1}=P_{N+1}=0$. Since only one ion can escape or enter at any one time, $P_{k}$ is coupled only to the neighbouring states $P_{k-1}$ and $P_{k+1}$. Note that $H_{N}=G_{N}=0$, since no ions can enter when the channel is fully occupied.

The first two terms (i.e. the first line) on the right-hand side of (2.3a) correspond to ion diffusion and ion drift respectively, where the drift term includes the external potential as well as ion-ion interactions. The third term corresponds to a new ion entering the $k$-ion channel from intracellular or extracellular solution; this term is negative since such an event leads to a transition from a $k$-ion channel to a $(k+1)$-ion channel. The fourth and fifth terms correspond to a new ion entering a $(k-1)$-ion channel from the left and right respectively. The sixth and seventh terms (i.e. the last line) of $(2.3 \mathrm{a})$ correspond to ions leaving a $(k+1)$-ion channel from the left and right respectively. 
The boundary conditions on 2.3a are

$$
\begin{gathered}
P_{k}\left(-L, x_{2}, \ldots, x_{k}\right)=0, \\
P_{k}\left(x_{1}, \ldots, x_{k-1}, L\right)=0,
\end{gathered}
$$

along with the no-flux condition on the interface $x_{i}=x_{i+1}$,

$$
\lim _{x_{i} \rightarrow x_{i+1}}\left(\frac{\partial P_{k}}{\partial x_{i}}-P_{k} \frac{1}{k_{B} T} f_{i}^{k}\right)=\lim _{x_{i} \rightarrow x_{i+1}}\left(\frac{\partial P_{k}}{\partial x_{i+1}}-P_{k} \frac{1}{k_{B} T} f_{i+1}^{k}\right)
$$

for $i=1, \ldots, k-1$, which ensures that the ions are correctly labelled. The reason we have had to write this as a limit is that the inter-ion potential tends to infinity as $x_{i} \rightarrow x_{i+1}$, while $P_{k}$ tends to zero. A local analysis shows that we need $P_{k}$ to tend to zero faster than $\left(x_{i+1}-x_{i}\right)^{2}$.

We note the following normalisation condition, which holds at all times $t$,

$$
\sum_{k=0}^{N} \int_{\Gamma_{k}} P_{k}\left(x_{1}, \ldots, x_{k}, t\right) d x_{1} \cdots d x_{k}=1,
$$

where $\Gamma_{k}$ is the available state space when there are $k$ ions in the channel, namely $\Gamma_{k}=$ $\left\{\left(x_{1}, \ldots, x_{k}\right): x_{1}<x_{2}<\cdots<x_{k}\right\}$. We will usually be interested in the steady state; in that case we solve the coupled hierarchical Fokker-Planck equations for the stationary probability distribution $\widetilde{P}_{k}=\lim _{t \rightarrow \infty} P_{k}(t)$ for $k=0,1, \ldots, N$.

2.2. An example with $N=2$. We exemplify the theory above with a simple channel that is selective to cations with elementary charge $e=1.6 \times 10^{-19} \mathrm{C}$. The selectivity of this type of channel is generally caused by negative charged boundary proteins, which decrease the energy barrier imposed by the narrow structure and assist the permeation of cations. For example, the oxygen atoms of four carbonyl groups in the selectivity filter of the potassium channel can be modelled by putting four negative partial charges equally spaced on a ring of radius $d$ that is perpendicular to the $x$-axis [9].

We consider the simplest possible example of multi-ion channel with capacity $N=2$ and a single binding site $M=1$. The binding site is located at the position $x=\xi$ and is a potential well generated by a ring of fixed partial negative charges a distance $d$ from the channel axis. By Coulomb's law, the potential energy $\Phi_{1}\left(x_{1}\right)$ seen by one cation at $x_{1}$ with charge $e$ traversing through the channel is

$$
\Phi_{1}\left(x_{1}\right)=\frac{e}{k_{B} T}\left(\frac{-k_{e} Z}{\sqrt{\left(x_{1}-\xi\right)^{2}+d^{2}}}+U x_{1}\right) .
$$

where $k_{e}$ the Coulomb force constant and $Z$ the total fixed charge on the ring, and $U$ is the constant field, which imposes a potential difference $2 U L$ across the channel $[-L, L]$. This potential difference is small compared to the potential well, and does not change the shape of the potential well but merely tilts it by a small angle. The force on the ion due to the potential is

$$
f_{1}^{1}=-k_{B} T \frac{\mathrm{d} \Phi_{1}}{\mathrm{~d} x_{1}}
$$

When there are two cations in the channel, at positions $x_{1}$ and $x_{2}$, the overall potential energy $\Phi_{2}\left(x_{1}, x_{2}\right)$, including the interaction between the two free ions, is

$$
\Phi_{2}\left(x_{1}, x_{2}\right)=\frac{e}{k_{B} T}\left(\frac{-k_{e} Z}{\sqrt{\left(x_{1}-\xi\right)^{2}+d^{2}}}+\frac{-k_{e} Z}{\sqrt{\left(x_{2}-\xi\right)^{2}+d^{2}}}+\frac{k_{e} e}{\left|x_{1}-x_{2}\right|}+U\left(x_{1}+x_{2}\right)\right) .
$$


The forces on the two ions are then

$$
f_{1}^{2}=-k_{B} T \frac{\partial \Phi_{2}}{\partial x_{1}}, \quad f_{2}^{2}=-k_{B} T \frac{\partial \Phi_{2}}{\partial x_{2}} .
$$

Finally we need to specify the entry rates $H_{k}$ and $G_{k}$ and entry distribution functions $h$ and $g$. We choose the simplest possible model for the entry distribution function. We suppose that the ions entering from the left are all placed at a position $x_{-}$near the left-hand end of the channel, while ions enterying from the right are placed at a position $x_{+}$near the right-hand end of the channel, that is

$$
h(x)=\delta\left(x-x_{-}\right), \quad g(x)=\delta\left(x-x_{+}\right) .
$$

We have to be careful in implementing this condition that we preserve the order of the ions in the channel. We choose to do this as follows: if we are attempting to place an ion at position $x_{-}$, and the position of the existing ion $x_{1}<x_{-}$, then we abandon the insertion of the new ion. A similar procedure is implemented at the right-hand end. In effect this means that the rate of entry is chosen to be zero whenever the position $x_{1}$ of the existing ion is such that $x_{1}<x_{-}$ or $x_{1}>x_{+}$. (An alternative procedure would be to modify the distribution functions $h$ and $g$ so that $h=0$ if $x_{1}<x_{-}$and $g=0$ if $x_{1}>x_{+}$, but this would mean altering them from the present $\delta$-functions.)

In general the entry rates may be functions of the current ion numbers and locations as well as the intracellular $\mathscr{I}$ and extracellular $\mathscr{E}$ environments. However, for this illustrative example we suppose that they are constant subject to the constraint set out above. Thus we choose

$$
H_{0}=\lambda, \quad G_{0}=\mu, \quad H_{1}=\lambda \Theta\left(x_{1}-x_{-}\right), \quad G_{1}=\mu \Theta\left(x_{+}-x_{1}\right)
$$

where $\Theta$ is the Heaviside function. Recall that $H_{2}=G_{2}=0$ since the channel is then fully occupied. To run the Brownian simulation, we set the time step $\Delta t=100 \mathrm{~ns}$, and the physical parameters as

$$
\begin{aligned}
L=1 \mathrm{~nm}, \quad x_{ \pm}= \pm 0.9 \mathrm{~nm}, \quad \xi=0 \mathrm{~nm}, \quad d=0.5 \mathrm{~nm}, \quad D=1 \mathrm{~nm}^{2} \cdot \mathrm{ns}^{-1}, \quad \lambda=\mu=5 \mathrm{~ns}^{-1}, \\
T=298 \mathrm{~K}, \quad k_{B}=1.38 \times 10^{-23} \mathrm{~J} \cdot \mathrm{K}^{-1}, \quad U=0 \mathrm{~V} \cdot \mathrm{nm}^{-1}, \quad Z=e .
\end{aligned}
$$

We use the nanometer as the unit of length and the nanosecond as the unit of time. We evolve (2.2) for $2 \times 10^{9}$ iterations until a dynamic equilibrium is reached. During the simulation the number of ions in the channel varies in time as ions enter and leave. We record the number of ions and their locations at each time step. We find that the proportion of time spent with $k$ ions in the channel, $J_{k}$ say, is given by

$$
J_{0} \approx 0.0000, \quad J_{1} \approx 0.8986, \quad J_{2} \approx 0.1014
$$

Thus, for these parameters, the channel is almost never empty, and for nearly $90 \%$ of the time there is just one ion in the channel, with two ions the remaining $10 \%$ of the time. The histograms of 2-ion distribution and 1-ion distribution are plotted in Fig. 2.3(a) and Fig. 2.4(a) respectively.

The stationary probability distributions $\widetilde{P}_{2}\left(x_{1}, x_{2}\right), \widetilde{P}_{1}\left(x_{1}\right)$ and $\widetilde{P}_{0}$ satisfy the stationary 


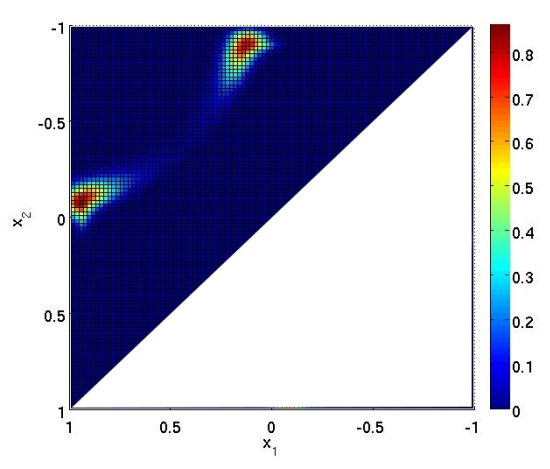

(a) Histogram

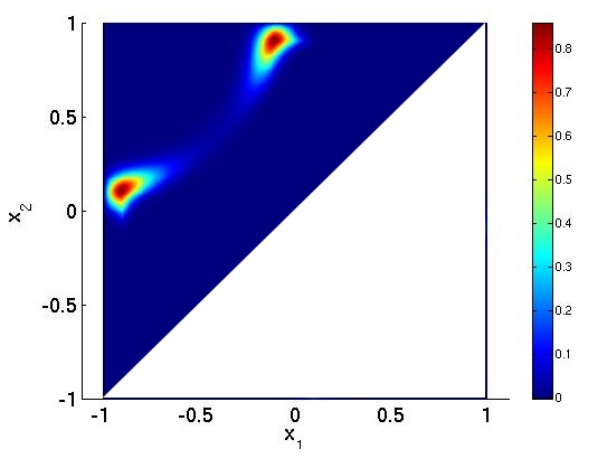

(b) $\widetilde{P}_{2}\left(x_{1}, x_{2}\right)$

FIG. 2.3. Using the parameters in 2.6, the stationary probability density of a 2-ion channel is computed as (a) histogram from Brownian dynamics simulation; (b) solution of $2.8 \mathrm{a}]-2.8 \mathrm{e}]$. Here $x_{1}$ is the position of the first ion and $x_{2}$ is the position of the second ion; since we label the ions such that $x_{1}<x_{2}$ the state space is a triangle.

Fokker-Planck equations for a two-ion channel, namely

$$
\begin{aligned}
0= & D \nabla \cdot\left(\nabla \widetilde{P}_{2}\left(x_{1}, x_{2}\right)+\widetilde{P}_{2}\left(x_{1}, x_{2}\right) \nabla \Phi_{2}\left(x_{1}, x_{2}\right)\right) \\
& +\lambda \Theta\left(x_{2}-x_{-}\right) \delta\left(x_{1}-x_{-}\right) \widetilde{P}_{1}\left(x_{2}\right)+\mu \Theta\left(x_{+}-x_{1}\right) \delta\left(x_{2}-x_{+}\right) \widetilde{P}_{1}\left(x_{1}\right) \\
0= & D \frac{\mathrm{d}}{\mathrm{d} x_{1}}\left(\frac{\mathrm{d} \widetilde{P}_{1}}{\mathrm{~d} x_{1}}\left(x_{1}\right)+\widetilde{P}_{1}\left(x_{1}\right) \frac{\mathrm{d} \Phi_{1}}{\mathrm{~d} x_{1}}\left(x_{1}\right)\right)-\left(\lambda \Theta\left(x_{1}-x_{-}\right)+\mu \Theta\left(x_{+}-x_{1}\right)\right) \widetilde{P}_{1}\left(x_{1}\right) \\
& +\lambda \delta\left(x_{1}-x_{-}\right) \widetilde{P}_{0}+\mu \delta\left(x_{1}-x_{+}\right) \widetilde{P}_{0} \\
& +D\left(\frac{\partial \widetilde{P}_{2}}{\partial x_{1}}+\widetilde{P}_{2} \frac{\partial \Phi_{2}}{\partial x_{1}}\right)\left(-L, x_{1}\right)-D\left(\frac{\partial \widetilde{P}_{2}}{\partial x_{2}}+\widetilde{P}_{2} \frac{\partial \Phi_{2}}{\partial x_{2}}\right)\left(x_{1}, L\right) \\
0= & -(\lambda+\mu) \widetilde{P}_{0}+D\left(\frac{\mathrm{d} \widetilde{P}_{1}}{\mathrm{~d} x_{1}}+\widetilde{P}_{1} \frac{\mathrm{d} \Phi_{1}}{\mathrm{~d} x_{1}}\right)(-L)-D\left(\frac{\mathrm{d} \widetilde{P}_{1}}{\mathrm{~d} x_{1}}+\widetilde{P}_{1} \frac{\mathrm{d} \Phi_{1}}{\mathrm{~d} x_{1}}\right)(L)
\end{aligned}
$$

with the boundary conditions

$$
\widetilde{P}_{2}\left(-L, x_{2}\right)=\widetilde{P}_{2}\left(x_{1}, L\right)=0, \quad \widetilde{P}_{1}(-L)=\widetilde{P}_{1}(L)=0 .
$$

and

$$
\lim _{x_{1} \rightarrow x_{2}}\left(\frac{\partial \widetilde{P}_{2}}{\partial x_{1}}+\widetilde{P}_{2} \frac{\partial \Phi_{2}}{\partial x_{1}}\right)=\lim _{x_{1} \rightarrow x_{2}}\left(\frac{\partial \widetilde{P}_{2}}{\partial x_{2}}+\widetilde{P}_{2} \frac{\partial \Phi_{2}}{\partial x_{2}}\right) .
$$

We solve 2.8a-2.8e by the finite element PDE solver Comsol with 28800 elements. The stationary distribution $\widetilde{P}_{2}\left(x_{1}, x_{2}\right)$ is shown in Fig. 2.3(b) and $\widetilde{P}_{1}\left(x_{1}\right)$ is shown in Fig. 2.4(b) We see that these agree with the histograms in Fig. 2.3(a) and Fig. 2.4(a) obtained from Brownian dynamics simulations.

We see that $\widetilde{P}_{2}\left(x_{1}, x_{2}\right)$ is localised around two discrete states near $\left(x_{-}, \xi\right)$ and $\left(\xi, x_{+}\right)$, while $\widetilde{P}_{1}\left(x_{1}\right)$ is localised around $x_{1}=\xi$. The most likely path between the two states of $\widetilde{P}_{2}\left(x_{1}, x_{2}\right)$ can also be faintly seen.

This localisation of $\widetilde{P}_{2}$ and $\widetilde{P}_{1}$ motivates the definition of a small number of discrete states which the system can adopt, which is the basis for the discrete transition rate theory described in the next section. 


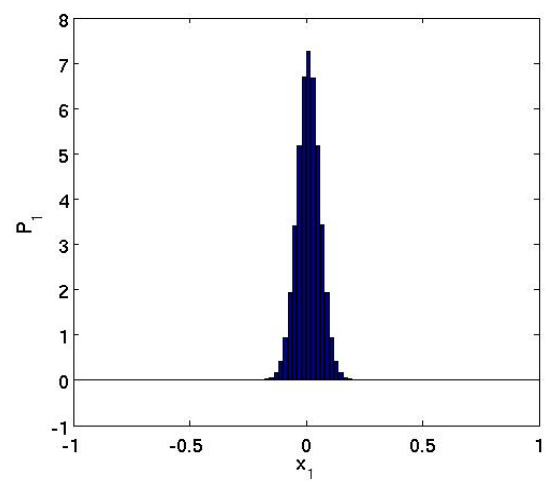

(a) Histogram

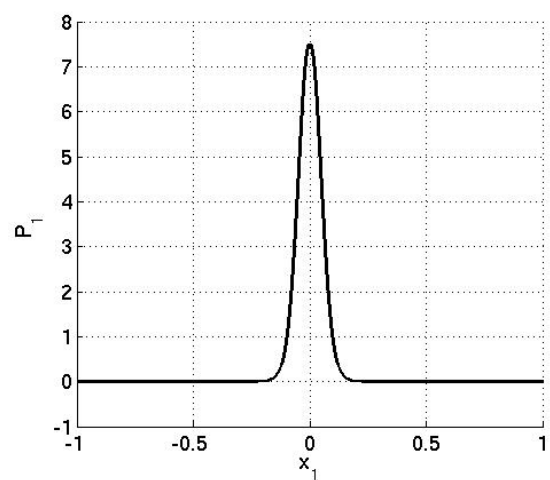

(b) $\widetilde{P}_{1}\left(x_{1}\right)$

FIG. 2.4. Using the parameters in 2.6, the stationary probability density of the one-ion state is computed by (a) histogram from Brownian dynamics simulation; (b) solution of 2.8a-2.8e. Here $x_{1}$ is the position of the single ion in the channel.

3. Discrete transition rate theory. We saw in our two-ion example (Figs. 2.3 and 2.4 that $\widetilde{P}_{2}$ was mainly localised around two regions in state space, while $\widetilde{P}_{1}$ was mainly localised around one region. Suppose, in general, that when there are $k$ ions in the channel the stationary probability distribution $\widetilde{P}_{k}\left(x_{1}, \ldots, x_{k}\right)$ is mainly localised around $L_{k}$ small regions. Let us denote these regions by

$$
S_{k}^{(i)} \subset \Gamma_{k} \quad \text { for } i=1, \ldots, L_{k} ;
$$

then $\widetilde{P}_{k}$ is very small outside $\cup_{i=1}^{L_{k}} S_{k}^{(i)}$, so that

$$
\int_{\Gamma_{k} \backslash \cup_{i=1}^{L_{k}} S_{k}^{(i)}} \widetilde{P}_{k}\left(x_{1}, \ldots, x_{k}\right) \mathrm{d} x_{1} \ldots \mathrm{d} x_{k} \approx 0
$$

The idea of discrete rate theory is to replace the continuous variable $\widetilde{P}_{k}$ with a set of discrete probabilities corresponding to the states $S_{k}^{(i)}$, so that

$$
\widetilde{P}_{k}^{(i)}=\int_{S_{k}^{(i)}} \widetilde{P}_{k}\left(x_{1}, \ldots, x_{k}\right) \mathrm{d} x_{1} \cdots \mathrm{d} x_{k}
$$

is the (stationary) probability that $\left(x_{1}, \ldots, x_{k}\right) \in S_{k}^{(i)}$. Note that $\widetilde{P}_{k}^{(i)}$ is just a number: it is independent of spatial variables. In total there are $L_{\Sigma}=\sum_{k=0}^{N} L_{k}$ states in the channel, and the sum of the probabilities of all $L_{\Sigma}$ states is unity according to (2.4), that is,

$$
\sum_{k=0}^{N} \sum_{i=1}^{L_{k}} \widetilde{P}_{k}^{(i)}=1
$$

We now imagine a Markov chain in which the channel undergoes transitions from one of these discrete states to another, with the transition probabilities dependent only on the current state (i.e. no past history is involved). This Markov chain is illustrated in Fig. 2.2. Such Markov chains for ion channels have been previously considered for a single ion in a manywell channel [1]. However, multiple occupancy of the channel leads to a more complicated transition structure. 
Since only one ion can enter or leave at once (so that $\widetilde{P}_{k}$ is coupled only to $\widetilde{P}_{k-1}$ and $\widetilde{P}_{k+1}$ ) we see that $S_{k}^{(i)}$ may have transitions to and from only the states $S_{k}^{(\cdot)}, S_{k-1}^{(\cdot)}$ and $S_{k+1}^{(\cdot)}$. The general master equation for the time-dependent probability $P_{k}^{(i)}(t)$ is of the form

$$
\begin{aligned}
\frac{\mathrm{d}}{\mathrm{d} t} P_{k}^{(i)}(t)= & \underbrace{\sum_{j} \alpha_{k-1}^{(i, j)} P_{k-1}^{(j)}(t)+\sum_{l} \beta_{k+1}^{(i, l)} P_{k+1}^{(l)}(t)+\sum_{m} \gamma_{k}^{(i, m)} P_{k}^{(m)}(t)}_{\text {influx }} \\
& -\underbrace{\left(\sum_{j} \alpha_{k}^{(j, i)}+\sum_{l} \beta_{k}^{(l, i)}+\sum_{m} \gamma_{k}^{(m, i)}\right) P_{k}^{(i)}(t)}_{\text {Outflux }},
\end{aligned}
$$

where $\alpha_{k}^{(i, j)}$ is the transition rate from $S_{k}^{(j)}$ to $S_{k+1}^{(i)}, \beta_{k}^{(i, j)}$ is the transition rate from $S_{k}^{(j)}$ to $S_{k-1}^{(i)}$, and $\gamma_{k}^{(i, j)}$ is the transition rate from $S_{k}^{(j)}$ to $S_{k}^{(i)}$. Thus $\alpha$ describes the influx of a new ion, $\beta$ describes the loss of an ion to the intracellular or extracellular environment, and $\gamma$ describes a hopping of the ions within the channel. In fact we expect many of these rates to be zero, since, for example, when we add a new ion to a channel it must occupy either the left-most or rightmost potential well.

The entry rates for new ions $\alpha_{k}^{(i, j)}$ may be determined from $H_{k}$ and $G_{k}$, which for the present purposes we are assuming are given. The ion escape rates $\beta_{k}^{(i, j)}$ and hopping rates $\gamma_{k}^{(i, j)}$ can be computed from the notation of mean escape time and splitting probability, as described below.

To define the mean escape time we set all the influx probabilities to zero. We then suppose that the channel initially contains $k$ ions located at positions $x_{1}, \ldots, x_{k}$. We define the mean escape time $\tau_{k}\left(x_{1}, \ldots, x_{k}\right)$ to be the average time before the channel undergoes a transition to a $(k-1)$-ion configuration, that is, the average time for one ion to leave the channel. Using the backward-Kolmogorov equation [20] it can be shown that $\tau_{k}$ satisfies

$$
\begin{gathered}
\Delta \tau_{k}-\nabla \Phi_{k} \cdot \nabla \tau_{k}=-\frac{1}{D}, \quad\left(x_{1}, \ldots, x_{k}\right) \in \Gamma_{k} \\
\tau_{k}=0 \text { if } x_{1}=-L \text { or } x_{k}=L .
\end{gathered}
$$

Then the mean escape time from state $S_{k}^{(i)}$ is given by

$$
\tau_{k}\left[S_{k}^{(i)}\right]=\frac{\int_{S_{k}^{(i)}} \tau_{k} P_{k} \mathrm{~d} x_{1} \cdots \mathrm{d} x_{k}}{\int_{S_{k}^{(i)}} P_{k} \mathrm{~d} x_{1} \cdots \mathrm{d} x_{k}} .
$$

We now determine a similar expression for $\tau_{k}\left[S_{k}^{(i)}\right]$ using the discrete transition rate model. Equating the two expressions will then provide information on the rates $\beta_{k}^{(i, j)}$ and $\gamma_{k}^{(i, j)}$.

To this end suppose that the channel is initially in the state $S_{k}^{(i)}$, so that $P_{k}^{(i)}(0)=1$, $P_{m}^{(j)}(0)=0$ otherwise. As before the influx rates $\alpha_{k}$ are set to be zero. The master equation (3.1) for $P_{k}^{(\cdot)}$ then decouples from those for $P_{k-1}^{(\cdot)}$ and $P_{k+1}^{(\cdot)}$, and we can solve for $P_{k}^{(i)}$. Given this solution we can determine the mean escape time $\tau_{k}\left[S_{k}^{(i)}\right]$ as

$$
\tau_{k}\left[S_{k}^{(i)}\right]=\frac{\sum_{l} \sum_{j} \int_{0}^{\infty} t \beta_{k}^{(l, j)} P_{k}^{(j)}(t) \mathrm{d} t}{\sum_{l} \sum_{j} \int_{0}^{\infty} \beta_{k}^{(l, j)} P_{k}^{(j)}(t) \mathrm{d} t} .
$$


In calculating the mean escape time we have not distinguished between the case that the first ion leaves from left end into intracellular electrolyte $\mathscr{I}$ and the case where the last ion leaves from right end into extracellular electrolyte $\mathscr{E}$. However, it is important that the discrete state model gets the ratio of these probabilities correct, since this is what causes a net ionic flux through the channel. Thus the second piece of information we use to determine the rates $\beta_{k}^{(i, j)}$ and $\gamma_{k}^{(i, j)}$ is the splitting probability $\rho_{k}\left(x_{1}, \ldots, x_{k}\right)$. This is defined to be the probability of the first ion to exit was $x_{1}$ from the left-hand side of the channel, under the condition that an ion-escaping event from a $k$-ion to a $(k-1)$-ion channel has occurred, given that the $k$ ions started in positions $\left(x_{1}, \ldots, x_{k}\right)$ initially. The splitting probability function $\rho_{k}$ satisfies,

$$
\begin{array}{ll}
\Delta \rho_{k}-\nabla \Phi_{k} \cdot \nabla \rho_{k}=0 & \text { for }\left(x_{1}, \ldots, x_{k}\right) \in \Gamma_{k} \\
\rho_{k}=1 \text { on } x_{1}=-L, & \rho_{k}=0 \text { on } x_{k}=L .
\end{array}
$$

As with $\tau_{k}$, we can now calculate the splitting probability for state $S_{k}^{(i)}$ as

$$
\rho_{k}\left[S_{k}^{(i)}\right]=\frac{\int_{S_{k}^{(i)}} \rho_{k} P_{k} \mathrm{~d} x_{1} \cdots \mathrm{d} x_{k}}{\int_{S_{k}^{(i)}} P_{k} \mathrm{~d} x_{1} \cdots \mathrm{d} x_{k}} .
$$

To calculate the splitting probability from the Markov chain we need to separate $\beta_{k}^{(l, j)}$ into two individual rates representing the case that an ion leaves to the right into the extracellular domain, and the case than an ion moves to the left into the intracellular domain, that is, we write

$$
\beta_{k}^{(l, j)}=\beta_{k}^{+(l, j)}+\beta_{k}^{-(l, j)} .
$$

Then the probability that an ion escapes to the left given that it escapes is

$$
\rho_{k}\left[S_{k}^{(i)}\right]=\frac{\sum_{l} \sum_{j} \int_{0}^{\infty} \beta_{k}^{-(l, j)} P_{k}^{(j)}(t) \mathrm{d} t}{\sum_{l} \sum_{j} \int_{0}^{\infty} \beta_{k}^{-(l, j)} P_{k}^{(j)}(t) \mathrm{d} t+\sum_{l} \sum_{j} \int_{0}^{\infty} \beta_{k}^{+(l, j)} P_{k}^{(j)}(t) \mathrm{d} t} .
$$

Note that, as in the case of the escape time $\tau_{k}$, the right-hand side depends on $S_{k}^{(i)}$ through the initial condition on $P_{k}^{(j)}$.

By equating (3.3) with 3.4 and 3.6 with 3.7 we have a number of equations to help determine the unknown rates $\beta_{k}^{(l, j)}$ and $\gamma_{k}^{(l, j)}$. Since only the left-most (respectively rightmost) ion can escape from the left-hand side of the channel (respectively right-hand side), many of the rates $\beta_{k}^{(l, j)}$ will infact be zero. If we still do not have enough equations to determine the remaining $\beta_{k}^{(l, j)}$ and $\gamma_{k}^{(l, j)}$, then it will be necessary to determine some of the transition rates between internal states. Since these do not involve a change in the number of ions in the channel, they may be determined by standard techniques.

Note that to determine the net flow of ions through the channel we will also have to distingiush between ion entry from the left and from the right, that is, we should also split

$$
\alpha_{k}^{(i, j)}=\alpha_{k}^{-(i, j)}+\alpha_{k}^{+(i, j)} .
$$

However, in most cases (at least) one of these rates will be zero, since it is not possible to have the same transition between two states occuring with an ion entering from either side. The one case where this is possible is the transition between an empty channel and a one-ion channel, which occurs in our example below. 


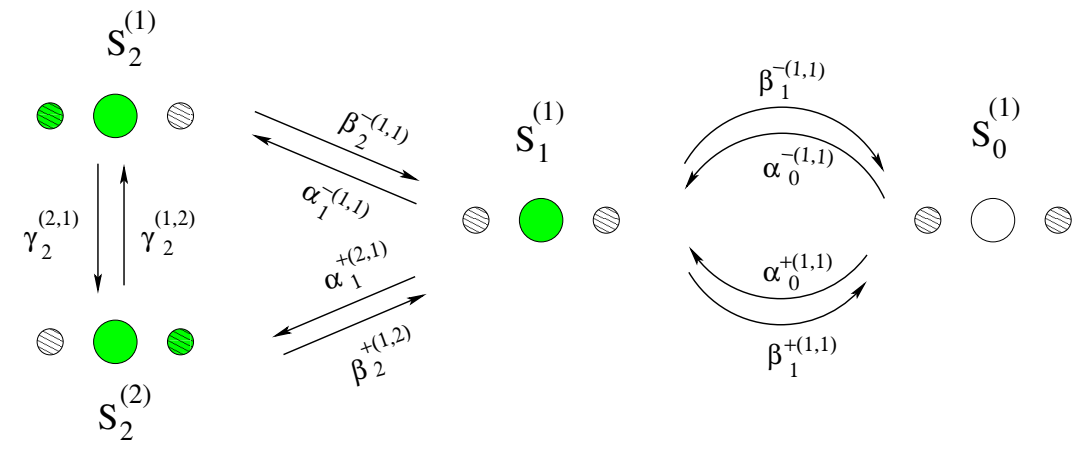

FIG. 3.1. The transitions between the four different states: the three circles represent the left entry point, the binding site and the right entry point; a (green) filled circle indicates the presence of an ion.

3.1. Example of two-ion channel. Now we revisit the example of two-ion channel in Section 2.2 and illustrate the rate theory using the parameters in 2.6.

We have seen that the channel can exist in a 2-ion, 1-ion or 0-ion state. From Fig. 2.4 we see that $\widetilde{P}_{1}\left(x_{1}\right)$ is localised around the single region $x_{1}=\xi$, so that there is only one metastable state with one ion in the channel. From Fig. 2.3 we see that $\widetilde{P}_{2}\left(x_{1}, x_{2}\right)$ is localised around the two states $\left(x_{-}, \xi\right)$ and $\left(\xi, x_{+}\right)$. Thus there are two metastable states with two ions in the channel. Thus our Markov chain comprises the four states

$$
S_{2}^{(1)}:\left\{\left(x_{-}, \xi\right)\right\}, \quad S_{2}^{(2)}:\left\{\left(\xi, x_{+}\right)\right\}, \quad S_{1}^{(1)}:\{\xi\}, \quad S_{0}^{(1)}:\{\} .
$$

Thus $L_{2}=2, L_{1}=1, L_{0}=1$ and overall there are $L_{\Sigma}=4$ states for this channel. These states, and the transitions between them, are illustrated in Fig. 3.1. The circle at center represents the binding site $x=\xi$, and two other circles represent the left and right entry positions $x=x_{ \pm}$. A (green) filled circle represents a position occupied by an ion. Note that for the transitions between $S_{0}^{(1)}$ and $S_{1}^{(1)}$ it is important to distinguish between ions entering and leaving from the right and from the left, so that we can calculate the net flow of ions through the channel.

Let us first consider the ion entry rates. We find

$$
\alpha_{1}^{+(1,1)}=0, \quad \alpha_{1}^{-(1,1)}=\lambda, \quad \alpha_{1}^{+(2,1)}=\mu, \quad \alpha_{1}^{-(2,1)}=0, \quad \alpha_{0}^{+(1,1)}=\mu, \quad \alpha_{0}^{-(1,1)}=\lambda .
$$

Note that the two zero values arise because the transition from $S_{1}^{(1)}$ to $S_{2}^{(1)}$ occurs via an ion entering from the left, while that from $S_{1}^{(1)}$ to $S_{2}^{(2)}$ occurs via an ion entering from the right. Note also that $\alpha_{2}^{(i, j)}=0$ for all $i, j$ since with two ions in the channel is already full to capacity.

Let us now consider the ion leaving rates $\beta_{k}^{(i, j)}$. In principle we have six of these to determine. However, since the transition from $S_{2}^{(1)}$ to $S_{1}^{(1)}$ must occur via an ion leaving from the left we know that $\beta_{2}^{+(1,1)}=0$. Similarly the transition from $S_{2}^{(2)}$ to $S_{1}^{(1)}$ must occur via an ion leaving from the right, so we know that $\beta_{2}^{-(1,2)}=0$. This leaves $\beta_{2}^{-(1,1)}, \beta_{2}^{+(1,2)}, \beta_{1}^{-(1,1)}$ and $\beta_{1}^{+(1,1)}$ to determine. To these we must add the two hopping rates $\gamma_{2}^{(1,2)}$ and $\gamma_{2}^{(2,1)}$.

Denoting the state of the system by the probability vector $\mathbf{P}=\left(P_{2}^{(1)}, P_{2}^{(2)}, P_{1}^{(1)}, P_{0}^{(1)}\right)^{T}$, the master equation governing the evolution of the Markov chain is then

$$
\frac{\mathrm{d} \mathbf{P}}{\mathrm{d} t}=\mathscr{T} \cdot \mathbf{P}(t),
$$


where the $4 \times 4$ transition matrix $\mathscr{T}$ is given by

$$
\mathscr{T}=\left(\begin{array}{cccc}
-\beta_{2}^{-(1,1)}-\gamma_{2}^{(2,1)} & \gamma_{2}^{(1,2)} & \alpha_{1}^{-(1,1)} & 0 \\
\gamma_{2}^{(2,1)} & -\beta_{2}^{+(1,2)}-\gamma_{2}^{(1,2)} & \alpha_{1}^{+(2,1)} & 0 \\
\beta_{2}^{-(1,1)} & \beta_{2}^{+(1,2)} & \begin{array}{c}
-\alpha_{1}^{-(1,1)}-\alpha_{1}^{+(2,1)} \\
-\beta_{1}^{+(1,1)}-\beta_{1}^{-(1,1)}
\end{array} & \alpha_{0}^{+(1,1)}+\alpha_{0}^{-(1,1)} \\
0 & 0 & \beta_{1}^{+(1,1)}+\beta_{1}^{-(1,1)} & -\alpha_{0}^{+(1,1)}-\alpha_{0}^{-(1,1)}
\end{array}\right) .
$$

As expected, the sum of each column of the matrix $\mathscr{T}$ is zero (since the system $(3.9 \mathrm{a})$ conserves probability), so the matrix is rank deficient. The stationary probability $\widetilde{\mathbf{P}}$ is the eigenvector associated with zero eigenvalue of matrix $\mathscr{T}$.

To calculate the mean escape time and splitting probability we set the all entry rates to zero and solve (3.9a). To emphasize that this is an auxilliary problem and not the true Markov chain we denote the probability of lying in each state by $q_{2}^{(1)}(t), q_{2}^{(2)}(t), q_{1}^{(1)}(t), q_{0}^{(1)}(t)$ respectively. Then $3.9 \mathrm{a}$ is

$$
\begin{aligned}
& \frac{\mathrm{d} q_{2}^{(1)}}{\mathrm{d} t}=-\left(\gamma_{2}^{(2,1)}+\beta_{2}^{-(1,1)}\right) q_{2}^{(1)}+\gamma_{2}^{(1,2)} q_{2}^{(2)}, \\
& \frac{\mathrm{d} q_{2}^{(2)}}{\mathrm{d} t}=-\left(\beta_{2}^{+(1,2)}+\gamma_{2}^{(1,2)}\right) q_{2}^{(2)}+\gamma_{2}^{(2,1)} q_{2}^{(1)}, \\
& \frac{\mathrm{d} q_{1}^{(1)}}{\mathrm{d} t}=\beta_{2}^{-(1,1)} q_{2}^{(1)}+\beta_{2}^{+(1,2)} q_{2}^{(2)}-\left(\beta_{1}^{+(1,1)}+\beta_{1}^{-(1,1)}\right) q_{1}^{(1)}, \\
& \frac{\mathrm{d} q_{0}^{(1)}}{\mathrm{d} t}=\left(\beta_{1}^{+(1,1)}+\beta_{1}^{-(1,1)}\right) q_{1}^{(1)} .
\end{aligned}
$$

The first two equations decouple. We start by considering the state $S_{2}^{(1)}$, that is, we solve 3.10a-3.10b subject to the initial conditions $q_{2}^{(1)}(0)=1$ and $q_{2}^{(2)}(0)=0$. This gives

$$
\begin{aligned}
\left(\begin{array}{c}
q_{2}^{(1)} \\
q_{2}^{(2)}
\end{array}\right)= & \frac{1}{\lambda_{1}-\lambda_{2}}\left(\begin{array}{c}
\lambda_{1}+\beta_{2}^{+(1,2)}+\gamma_{2}^{(1,2)} \\
\gamma_{2}^{(2,1)}
\end{array}\right) \exp \left(\lambda_{1} t\right) \\
& +\frac{1}{\lambda_{2}-\lambda_{1}}\left(\begin{array}{c}
\lambda_{2}+\beta_{2}^{+(1,2)}+\gamma_{2}^{(1,2)} \\
\gamma_{2}^{(2,1)}
\end{array}\right) \exp \left(\lambda_{2} t\right),
\end{aligned}
$$

where $\lambda_{1}, \lambda_{2}$ are two eigenvalues satisfying

$$
\begin{aligned}
\lambda_{1}+\lambda_{2} & =-\left(\beta_{2}^{-(1,1)}+\beta_{2}^{+(1,2)}+\gamma_{2}^{(2,1)}+\gamma_{2}^{(1,2)},\right) \\
\lambda_{1} \lambda_{2} & =\beta_{2}^{-(1,1)} \beta_{2}^{+(1,2)}+\beta_{2}^{-(1,1)} \gamma_{2}^{(1,2)}+\beta_{2}^{+(1,2)} \gamma_{2}^{(2,1)} .
\end{aligned}
$$

Using [3.4), the mean escape time $\tau_{2}\left[S_{2}^{(1)}\right]$ is

$$
\begin{aligned}
\tau_{2}\left[S_{2}^{(1)}\right] & =\frac{\int_{0}^{\infty} t\left(\beta_{2}^{-(1,1)} q_{2}^{(1)}(t)+\beta_{2}^{+(1,2)} q_{2}^{(2)}(t)\right) \mathrm{d} t}{\int_{0}^{\infty} \beta_{2}^{-(1,1)} q_{2}^{(1)}(t)+\beta_{2}^{+(1,2)} q_{2}^{(2)}(t) \mathrm{d} t} \\
& =\frac{\beta_{2}^{+(1,2)}+\gamma_{2}^{(2,1)}+\gamma_{2}^{(1,2)}}{\beta_{2}^{+(1,2)} \gamma_{2}^{(2,1)}+\beta_{2}^{-(1,1)} \gamma_{2}^{(1,2)}+\beta_{2}^{-(1,1)} \beta_{2}^{+(1,2)}},
\end{aligned}
$$


and, using 3.7 , the splitting probability $\rho_{2}\left[S_{2}^{(1)}\right]$ is

$$
\begin{aligned}
\rho_{2}\left[S_{2}^{(1)}\right] & =\frac{\int_{0}^{\infty} \beta_{2}^{-(1,1)} q_{2}^{(1)}(t) \mathrm{d} t}{\int_{0}^{\infty} \beta_{2}^{-(1,1)} q_{2}^{(1)}(t)+\beta_{2}^{+(1,2)} q_{2}^{(2)}(t) \mathrm{d} t} \\
& =\frac{\beta_{2}^{-(1,1)} \beta_{2}^{+(1,2)}+\beta_{2}^{-(1,1)} \gamma_{2}^{(1,2)}}{\beta_{2}^{-(1,1)} \beta_{2}^{+(1,2)}+\beta_{2}^{-(1,1)} \gamma_{2}^{(1,2)}+\beta_{2}^{+(1,2)} \gamma_{2}^{(2,1)}} .
\end{aligned}
$$

Similarly, by applying the initial conditions $q_{2}^{(1)}(0)=0$ and $q_{2}^{(2)}(0)=1$, we find

$$
\begin{aligned}
\tau_{2}\left[S_{2}^{(2)}\right] & =\frac{\beta_{2}^{-(1,1)}+\gamma_{2}^{(2,1)}+\gamma_{2}^{(1,2)}}{\beta_{2}^{+(1,2)} \gamma_{2}^{(2,1)}+\beta_{2}^{-(1,1)} \gamma_{2}^{(1,2)}+\beta_{2}^{-(1,1)} \beta_{2}^{+(1,2)},} \\
1-\rho_{2}\left[S_{2}^{(2)}\right] & =\frac{\beta_{2}^{-(1,1)} \beta_{2}^{+(1,2)}+\beta_{2}^{+(1,2)} \gamma_{2}^{(2,1)}}{\beta_{2}^{-(1,1)} \beta_{2}^{+(1,2)}+\beta_{2}^{-(1,1)} \gamma_{2}^{(1,2)}+\beta_{2}^{+(1,2)} \gamma_{2}^{(2,1)}} .
\end{aligned}
$$

Finally we have to consider the escape time and splitting probability for state $S_{1}^{(1)}$. With the initial condition $q_{2}^{(1)}(0)=q_{2}^{(2)}(0)=q_{0}^{(1)}(0)=0, q_{1}^{(1)}(0)=1$, equation $3.10 \mathrm{c}$ decouples and is easily solved to give

$$
\begin{aligned}
\tau_{1}\left[S_{1}^{(1)}\right] & =\frac{\int_{0}^{\infty} t q_{1}^{(1)}(t) \mathrm{d} t}{\int_{0}^{\infty} q_{1}^{(1)} \mathrm{d} t}=\frac{1}{\beta_{1}^{-(1,1)}+\beta_{1}^{+(1,1)}}, \\
\rho_{1}\left[S_{1}^{(1)}\right] & =\frac{\beta_{1}^{-(1,1)}}{\beta_{1}^{-(1,1)}+\beta_{1}^{+(1,1)}} .
\end{aligned}
$$

The mean escape times $\tau_{2}, \tau_{1}$ and the splitting probability $\rho_{2}, \rho_{1}$ can be obtained by solving (3.2) and (3.5), respectively with $k=2,1$ by Comsol. Fixing $U=0, \xi=0$, the mean escape time $\tau_{2}$ in triangular domain $\Gamma_{2}$ is plotted in Fig. 3.2(a) the splitting probability $\rho_{2}$ in $\Gamma_{2}$ is plotted in Fig. 3.2(b) Since there is no applied field across the channel $(U=0)$, and the potential well is at the center, the external potential is symmetric with $x=0$, therefore both functions are symmetric with $x_{1}+x_{2}=0$. We take the values of functions at the centre of the different states and obtain

$$
\begin{gathered}
\tau_{2}\left[S_{2}^{(1)}\right]=\tau_{2}\left(x_{-}, \xi\right)=0.01113, \quad \rho_{2}\left[S_{2}^{(1)}\right]=\rho_{2}\left(x_{-}, \xi\right)=0.9964, \\
\tau_{2}\left[S_{2}^{(2)}\right]=\tau_{2}\left(\xi, x_{+}\right)=0.01113, \quad \rho_{2}\left[S_{2}^{(2)}\right]=\rho_{2}\left(\xi, x_{+}\right)=0.0036, \\
\tau_{1}\left[S_{1}^{(1)}\right]=\tau_{1}(\xi)=3.9385 \times 10^{3}, \quad \rho_{1}\left[S_{1}^{(1)}\right]=\rho_{1}(\xi)=0.5 .
\end{gathered}
$$

Equating these expressions to those of (3.12) we find six equations for the six unknown rates. Solving these we find

$$
\beta_{2}^{-(1,1)}=\beta_{2}^{+(1,2)}=89.8283, \gamma_{2}^{(2,1)}=\gamma_{2}^{(1,2)}=0.3361, \beta_{1}^{-(1,1)}=\beta_{1}^{+(1,1)}=1.2695 \times 10^{-4} .
$$

Note that by using the mean escape time and the splitting probability we have not had to estimate the internal hopping rates $\gamma_{2}$ from the Fokker-Planck equation, but have been able to determine them from the auxillary problems we have solved. We will see in Section 5 that this is especially useful when the internal states are not so well defined. 


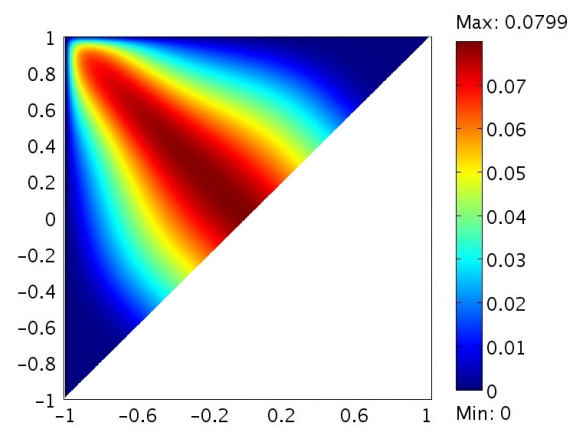

(a) $\tau_{2}\left(x_{1}, x_{2}\right)$

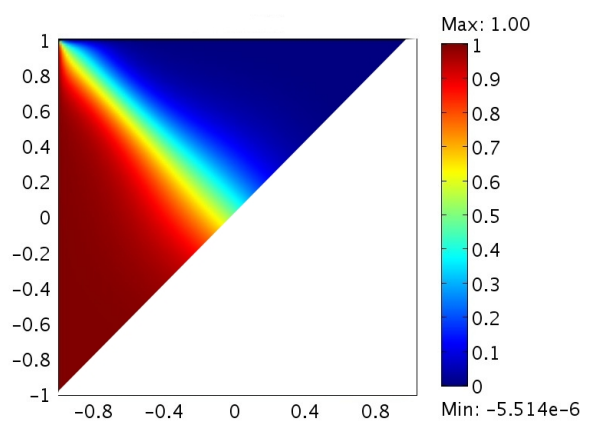

(b) $\rho_{2}\left(x_{1}, x_{2}\right)$

FIG. 3.2. The parameters are given in 2.6. (a) Mean escape time $\tau_{2}\left(x_{1}, x_{2}\right)$ for 2-ion transiting into 1-ion state. (b) Splitting probability $\rho_{2}\left(x_{1}, x_{2}\right)$ of ion exiting from left side under the condition that an ion escaping event occurs.

Since there is no external potential gradient in this case $(U=0)$ the rates are symmetric, and there is no net flux through the channel. However, we can already make some observations. Firstly, the rates $\beta_{1}^{ \pm(1,1)}$ are tiny compared to the others. Thus the channel will switch between single and double occupancy, but will almost never be empty of ions. We will confirm this when we consider the equilibrium occupancy of the channel in the next section. Secondly, the exit rates $\beta_{2}^{-(1,1)}$ and $\beta_{2}^{+(1,2)}$ are about 270 times as large as the hopping rates $\gamma_{2}^{(2,1)}$ and $\gamma_{2}^{(1,2)}$ and. This means that, for these values of the parameters, an incoming ion enters and leaves about 270 times before it manages to replace the bound ion in the potential well at the centre of the channel.

3.2. Stationary probability of each state. Now that we have determined all the transition rates in Fig. 3.1, the stationary probability for the number of ions in the channel can be calculated explicitly as

$$
\begin{aligned}
\widetilde{P}_{2}^{(1)}+\widetilde{P}_{2}^{(2)} & =\frac{\alpha_{1}^{-(1,1)} \tau_{2}\left[S_{2}^{(1)}\right]+\alpha_{1}^{+(2,1)} \tau_{2}\left[S_{2}^{(2)}\right]}{1+\frac{1}{\tau_{1}\left[S_{1}^{(1)}\right]} \frac{1}{\alpha_{0}^{-(1,1)}+\alpha_{0}^{+(1,1)}}+\alpha_{1}^{-(1,1)} \tau_{2}\left[S_{2}^{(1)}\right]+\alpha_{1}^{+(2,1)} \tau_{2}\left[S_{2}^{(2)}\right]} \approx 0.1002, \\
\widetilde{P}_{1}^{(1)} & =\frac{1}{1+\frac{1}{\tau_{1}\left[S_{1}^{(1)}\right]} \frac{1}{\alpha_{0}^{-(1,1)}+\alpha_{0}^{+(1,1)}}+\alpha_{1}^{-(1,1)} \tau_{2}\left[S_{2}^{(1)}\right]+\alpha_{1}^{+(2,1)} \tau_{2}\left[S_{2}^{(2)}\right]} \approx 0.8998, \\
\widetilde{P}_{0}^{(1)} & =\frac{\frac{1}{\tau_{1}\left(S_{1}^{(1)}\right)} \frac{1}{\alpha_{0}^{-(1,1)}+\alpha_{0}^{+(1,1)}}}{1+\frac{1}{\tau_{1}\left(S_{1}^{(1)}\right)} \frac{1}{\alpha_{0}^{-(1,1)}+\alpha_{0}^{+(1,1)}}+\alpha_{1}^{-(1,1)} \tau_{2}\left(S_{2}^{(1)}\right)+\alpha_{1}^{+(2,1)} \tau_{2}\left(S_{2}^{(2)}\right)} \approx 2.285 \times 10^{-5} .
\end{aligned}
$$

We see that these agree well with the probabilities obtained from a Brownian dynamics simulation in 2.7). The same probabilities may be obtained by integrating the solutions of FokkerPlanck equations over the configuration space, which gives

$$
\begin{aligned}
& I_{2} \equiv \int_{-L}^{L} \int_{-L}^{x_{2}} \widetilde{P}_{2}\left(x_{1}, x_{2}\right) \mathrm{d} x_{1} \mathrm{~d} x_{2} \approx 0.1001, \\
& I_{1} \equiv \int_{-L}^{L} \widetilde{P}_{1}\left(x_{1}\right) \mathrm{d} x_{1} \approx 0.8991, \quad I_{0} \equiv \widetilde{P}_{0} \approx 8.3 \times 10^{-4} .
\end{aligned}
$$


4. Current-voltage curve. The most important characteristic of an ion channel is its conductance. In this section, we investigate the channel conductance by examining the current-voltage curve for various values of the channel parameters.

The potential difference across a channel is usually around $100 \sim 200 \mathrm{mV}$. We therefore vary the potential gradient $U$ in the range $[-0.1,0.1] \mathrm{V} \mathrm{nm}^{-1}$, which gives a voltage drop in the range $[-200,200] \mathrm{mV}$ for a channel of length $2 \mathrm{~nm}$. Following the framework in Section 3. we compute the transition rates corresponding to each given value of $U$.

By examining the transition network in Fig. 3.1 we see that there are two different paths which lead to ions moving from the intracellular (left-hand side) to the extracellular (righthand side) domain, namely

PATH $1: S_{1}^{(1)} \stackrel{\alpha_{1}^{-(1,1)}}{\longrightarrow} S_{2}^{(1)} \stackrel{\gamma_{2}^{(2,1)}}{\longrightarrow} S_{2}^{(2)} \stackrel{\beta_{2}^{+(1,2)}}{\longrightarrow} S_{1}^{(1)}, \quad$ PATH 2: $S_{1}^{(1)} \stackrel{\beta_{1}^{-(1,1)}}{\longrightarrow} S_{0}^{(1)} \stackrel{\alpha_{0}^{-(1,1)}}{\longrightarrow} S_{1}^{(1)}$.

Both paths start with a channel with one ion bound at the potential well. In Path 1 another ion first enters the channel from the left-hand source to produce a two-ion channel. The two ions then hop to the right so the new ion lies in the potential well at the centre of the channel. The ion released from this well then exits the channel at the right. Thus in Path 1 we can think of a new ion coming in and knocking the present ion out the other side. In Path 2 the ion in the channel first leaves from the right to leave an empty channel, and then a new ion enters from the left.

By considering the transition rates we can determine the relative importance of each of these mechanisms. For the parameters in $\sqrt{2.6}$ the rate $\beta_{1}^{-(1,1)}$ is tiny and Path 1 dominates the current. Note that at equilibrium the ion flux entering from the left is balanced by the flux which leaves from right for each path, so that

$$
\alpha_{1}^{-(1,1)} \widetilde{P}_{1}^{(1)}=\beta_{2}^{+(1,2)} \widetilde{P}_{2}^{(2)}, \quad \alpha_{0}^{-(1,1)} \widetilde{P}_{0}^{(1)}=\beta_{1}^{-(1,1)} \widetilde{P}_{1}^{(1)} .
$$

Similarly ions flow from right to left via the paths,

PATH 3: $S_{1}^{(1)} \stackrel{\alpha_{1}^{+(2,1)}}{\longrightarrow} S_{2}^{(2)} \stackrel{\gamma_{2}^{(1,2)}}{\longrightarrow} S_{2}^{(1)} \stackrel{\beta_{2}^{-(1,1)}}{\longrightarrow} S_{1}^{(1)}, \quad$ PATH $4: S_{1}^{(1)} \stackrel{\beta_{1}^{+(1,1)}}{\longrightarrow} S_{0}^{(1)} \stackrel{\alpha_{0}^{+(1,1)}}{\longrightarrow} S_{1}^{(1)}$, and in equilibrium

$$
\alpha_{1}^{+(2,1)} \widetilde{P}_{1}^{(1)}=\beta_{2}^{-(1,1)} \widetilde{P}_{2}^{(1)}, \quad \alpha_{0}^{+(1,1)} \widetilde{P}_{0}^{(1)}=\beta_{1}^{+(1,1)} \widetilde{P}_{1}^{(1)} .
$$

Combining the current from each path the net current is given by

$$
\begin{aligned}
I & =e\left(\alpha_{0}^{-(1,1)} \widetilde{P}_{0}^{(1)}+\alpha_{1}^{-(1,1)} \widetilde{P}_{1}^{(1)}-\beta_{1}^{+(1,1)} \widetilde{P}_{1}^{(1)}-\beta_{2}^{-(1,1)} \widetilde{P}_{2}^{(1)}\right) \\
& =e\left(\beta_{1}^{-(1,1)} \widetilde{P}_{1}^{(1)}+\beta_{2}^{+(1,2)} \widetilde{P}_{2}^{(2)}-\alpha_{0}^{+(1,1)} \widetilde{P}_{0}^{(1)}-\alpha_{1}^{+(2,1)} \widetilde{P}_{1}^{(1)}\right),
\end{aligned}
$$

Next we study how the conductance of the channel varies with the external potential energy parameter $d / L$ and the dimensionless entry rates $L^{2} \lambda / D$ and $\mu L^{2} / D$. We plot in Fig. 4.1 the current-voltage (I-V) curve for different entry rates when $d / L=0.5$. For large voltages the current saturates since it is limited by the entry rate of ions $\lambda$ and $\mu$; this effect is illustrated in Fig. 4.1(b), In Fig. 4.2 we plot the I-V curve for various values of $d$ with a fixed entry rate. The slopes of these curves give the conductance of channel, which grows initially with increasing voltage until diminishing as saturation sets in. We see that as the dimensionless entry rate increases, or the potential well gets shallower, the conductance of the channel increases. 


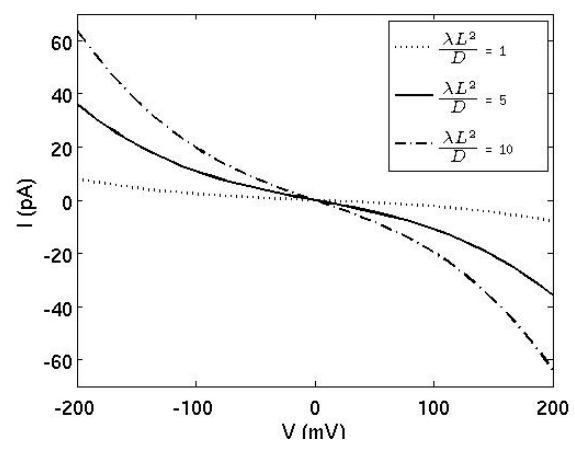

(a)

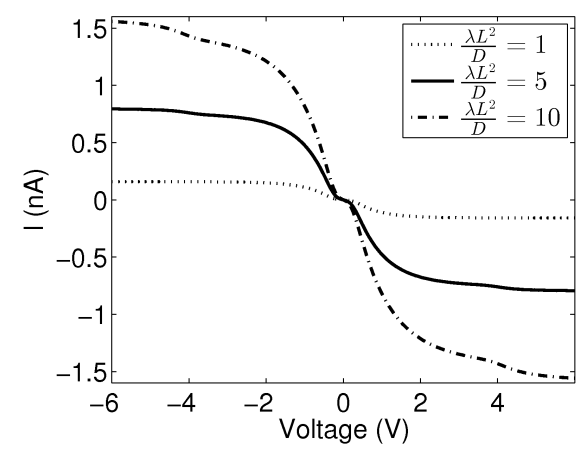

(b)

FIG. 4.1. I-V curve for $L^{2} \lambda / D=L^{2} \mu / D=1,5,10$ with $d / L=0.5$; all other parameters are as in 2.6. (a) for small voltages, for which internal transitions in the channel are rate limiting; (b) for larger voltages, at which saturation occurs due to the finite rate of entry of ions from the bulk.

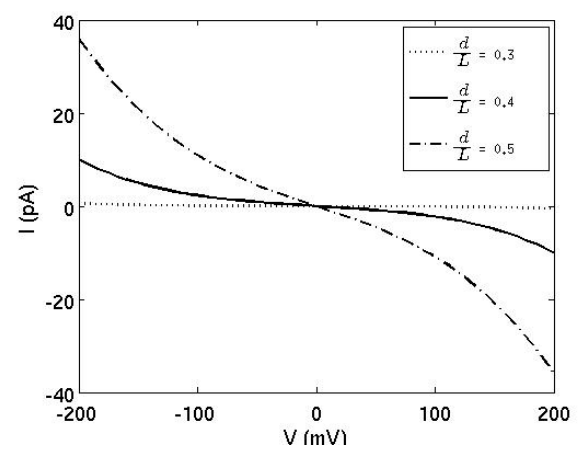

FIG. 4.2. $I$-V curve for $d / L=0.3,0.4,0.5$ with $L^{2} \lambda / D=L^{2} \mu / D=5$. All other parameters are as in 2.6.

5. Optimal geometry of potential. We showed that a channel with shallower potential well has larger conductivity in Fig. 4.2 if all other physical parameters are fixed. However, the depth of the potential well is not the only factor that determines the ion flux. Abad et al [1] studied the flux through a channel of capacity $N=1$ with symmetric M-shape potential energy, and showed that there exists a critial ratio $\sigma$ of the width of potential well over the length of channel, at which the flux is maximized. This optimal geometry of potential energy requires the potential well is neither too narrow $(\sigma \rightarrow 0)$ nor too wide $(\sigma \rightarrow 1)$.

We now perform a similar analysis of the multi-ion channel. For our $N=2$ example we study how the shape of an external potential on the protein boundary affects the conductivity of the channel. We vary the distance $d$ and carefully choose the external charge as

$$
Z=e \frac{2-\left(1+0.5^{2}\right)^{-0.5}}{d^{-1} L-\left(1+d^{2} L^{-2}\right)^{-0.5}},
$$

so that the depths of the resulting potential wells are all the same (and so that $Z=e$, when $d / L=0.5$ ); the variation of the potential well with $d$ is shown in Fig. 5.1(b) The resulting group of potential wells with applied field $U=-0.05 \mathrm{~V} \mathrm{~nm}^{-1}$ are plotted in Fig. 5.1(a), the darker curve corresponds to $Z=e, d=0.5 L$, the dotted curve shows how the potential wells 


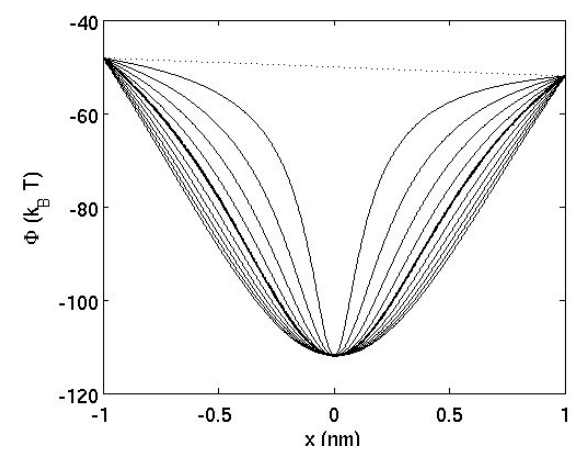

(a) $\Phi$ vs. $x$

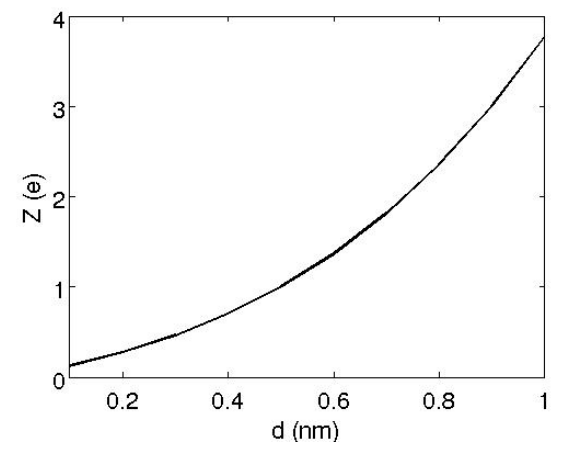

(b) $Z$ vs. $d$

FIG. 5.1. (a) A group of potential energy wells for $d=0.1,0.2,0.3, \ldots, 0.9,1.0 \mathrm{~nm}$ are plotted by solid curves respectively from narrow to wide well, the darker curve corresponds to $Z=e, d / L=0.5$, and the dotted curve corresponds the energy drop due to applied field $U=-0.05 \mathrm{~V} \mathrm{~nm}^{-1}$. (b) The external charge $Z$ as a function of $d$.

tilt with the applied field. Obviously, when $d$ is small, we have a steep potential drop near the binding site, and a relatively flat energy landscape near the ends of the channel. When $d / L$ gets large, the potential well tends to a curve that is steeper near the end of the channel and flatter near the binding site.

Now we fix $U=-0.05 \mathrm{~V} \mathrm{~nm}^{-1}, \lambda=\mu=5 \mathrm{~ns}^{-1}$, and plot in Fig. 5.2 the stationary probability density function $\widetilde{P}_{2}\left(x_{1}, x_{2}\right)$ obtained by solving stationary Fokker-Planck equations numerically for various values of $d$. Recall that since $x_{1}<x_{2}$, we only look at the upper left triangular domain. We observe that when the potential well is very narrow and steep at the binding site with $d=0.1 \mathrm{~nm}$, the probability distribution of two ions is localized at two tiny spots around $\left(x_{-}, \xi\right)$ and $\left(\xi, x_{+}\right)$. As $d$ increases until $d=0.5 \mathrm{~nm}$, the two spots grow a little larger and shift slightly away from $\left(x_{-}, \xi\right)$ and $\left(\xi, x_{+}\right)$. Thus for $d \in[0.1,0.5] \mathrm{nm}$ we can justify the use of our four-state rate theory. For $d$ between $0.5 \mathrm{~nm}$ and $0.6 \mathrm{~nm}$, a ridge of high probability distribution emerges that connects previous two spots at its two tails. This implies that instead of the two ions being trapped at one of two states, the two can wander back and forth freely between these two states. As $d$ increases even further to $d=0.9 \mathrm{~nm}$, the ridge shrinks to its center peak, which corresponds to the two ions both sitting in the potential well. Thus, for larger values of $d$, we have effectively only one state for the two-ion occupied channel.

Thus, for large $d$, we can define a single-chained three-state system

$$
S_{2}^{(1)}:\left\{\left(\left(x_{-}+\xi\right) / 2,\left(\xi+x_{+}\right) / 2\right)\right\}, \quad S_{1}^{(1)}:\{\xi\}, \quad S_{0}^{(1)}:\{\},
$$

which is similar to four-state system in Fig. 3.1, except that $S_{2}^{(1)}=S_{2}^{(2)}$ are combined and there is no hopping $\gamma_{2}^{(1,2)}$ and $\gamma_{2}^{(2,1)}$ between them. Following the framework in Section 3 . all the entry rates $\alpha_{0}^{-(1,1)}=\alpha_{1}^{-(1,1)}=\mu$ and $\alpha_{0}^{+(1,1)}=\alpha_{1}^{+(1,1)}=\lambda$ are prescribed, and the escaping rates are easily calculated as

$$
\beta_{k}^{-(1,1)}=\frac{\rho_{k}\left(S_{k}^{(1)}\right)}{\tau_{k}\left(S_{k}^{(1)}\right)}, \quad \beta_{k}^{+(1,1)}=\frac{1-\rho_{k}\left(S_{k}^{(1)}\right)}{\tau_{k}\left(S_{k}^{(1)}\right)}, \quad k=1,2 .
$$

In Fig. 5.3(a), we plot the mean escape time $\tau_{2}$ from the left state $S_{2}^{(1)}=(-0.9,0)$ (black solid curve) and the right state $S_{2}^{(2)}=(0,0.9)$ (black dash-dotted curve) in four-state formulation, 


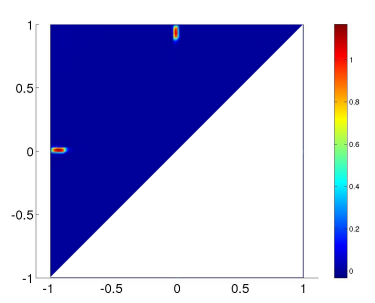

(a) $d=0.1 \mathrm{~nm}$

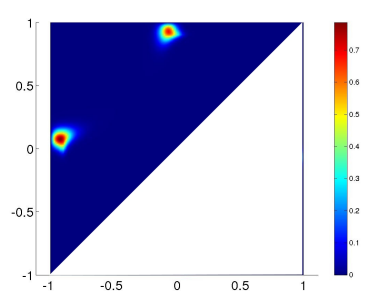

(d) $d=0.4 \mathrm{~nm}$

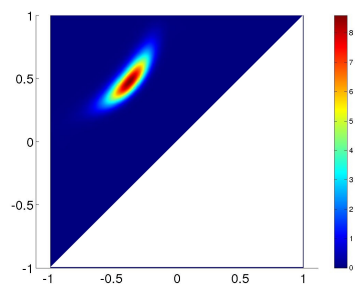

(g) $d=0.7 \mathrm{~nm}$

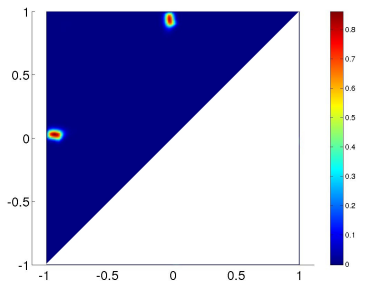

(b) $d=0.2 \mathrm{~nm}$

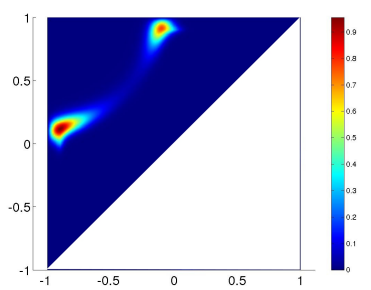

(e) $d=0.5 \mathrm{~nm}$

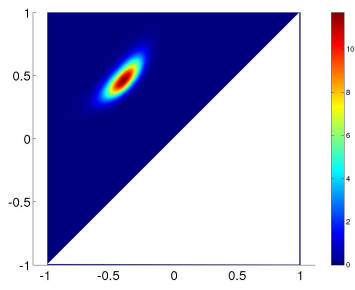

(h) $d=0.8 \mathrm{~nm}$

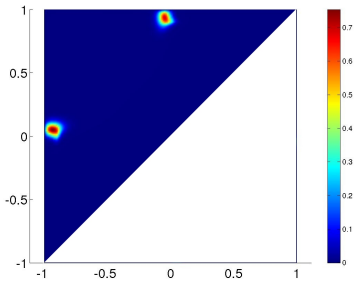

(c) $d=0.3 \mathrm{~nm}$

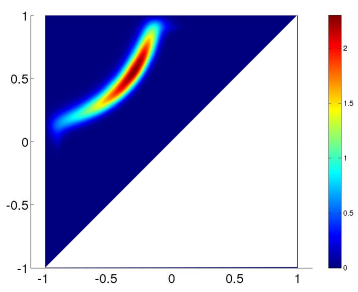

(f) $d=0.6 \mathrm{~nm}$

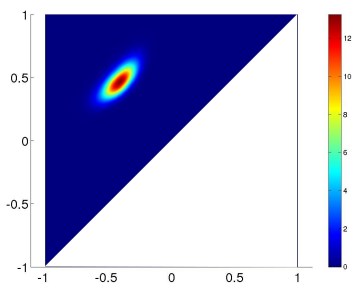

(i) $d=0.9 \mathrm{~nm}$

FIG. 5.2. Fixing $\lambda=\mu=5 \mathrm{~ns}^{-1}, U=-0.05 \mathrm{Vnm}^{-1}$, we plot the stationary probability distribution $\widetilde{P}_{2}(x, y)$ for $d=0.1,0.2,0.3,0.4,0.5,0.6,0.7,0.8,0.9 \mathrm{~nm}$, which are obtained by solving [2.8 numerically. It shows the change from two distinct states at $(-0.9,0)$ and $(0,0.9)$ to a ridge of high probability regime to one distinct state near $(-0.45,0.45)$, with the change of geometry of potential well.

and compare them with $\tau_{2}$ from the balanced state $S_{2}^{(1)}=(-0.45,0.45)$ (red solid curve) in the three-state formulation 5.1. Since the potential well is broader as $d$ increases, the second ion (the one which is not trapped in the well) feels its effect more, resulting in an exponential increase in the mean escape time. In a channel with descending voltage from left to right $\left(U=-0.05 \mathrm{~V} \mathrm{~nm}^{-1}\right)$, it takes a longer time for two ions in the left state $(-0.9,0)$ to escape than two ions in the right state $(0,0.9)$, so the black solid curve is above the black dashed curve. For two ions in the balanced state $S_{2}^{(1)}=(-0.45,0.45)$ in the middle of channel, it takes an even longer time, so the red solid curve is above the two black curves.

Notice that the ratio $\tau_{2}(-0.9,0) / \tau_{2}(0,0.9)$ is largest when $0.5<d<0.6$. Note also that for small $d$ the three-state formulation is invalid (so $\tau_{2}(-0.45,0.45)$ is meaningless) but that for large $d$ the ratio $\tau_{2}(-0.45,0.45) / \tau_{2}(-0.9,0)$ is close to 1 : for a broad potential the mean escape time is insensitive to the precise initial position of the two ions.

In Fig. 5.3(b) we plot the left splitting probability $\rho_{2}$ at the left state $S_{2}^{(1)}=(-0.9,0)$ (black solid curve) and the right splitting probability $1-\rho_{2}$ at the right state $S_{2}^{(2)}=(0,0.9)$ 


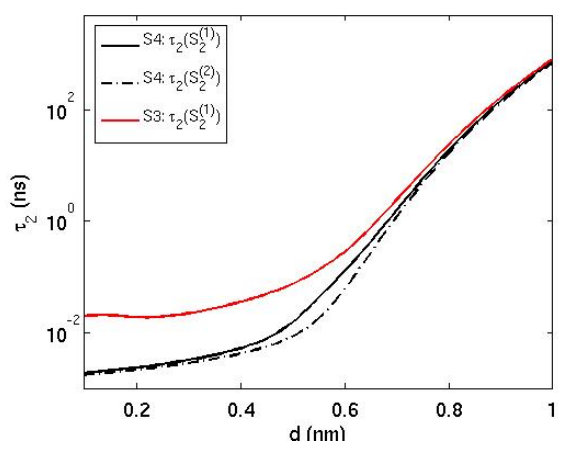

(a)

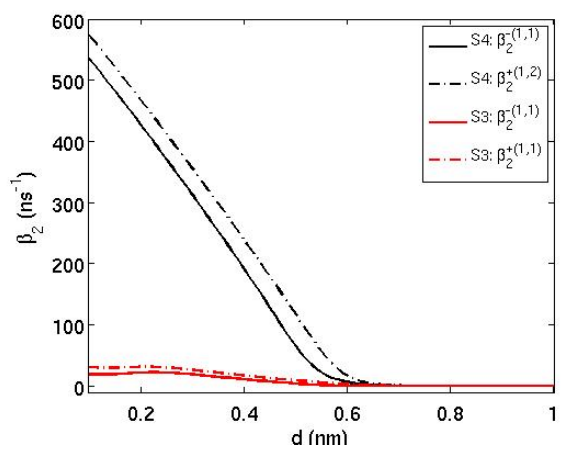

(c)

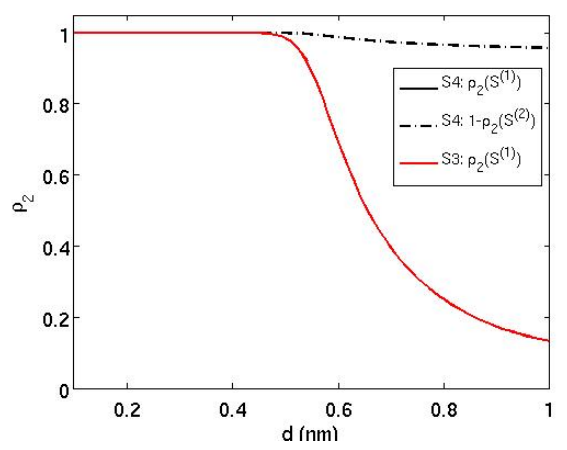

(b)

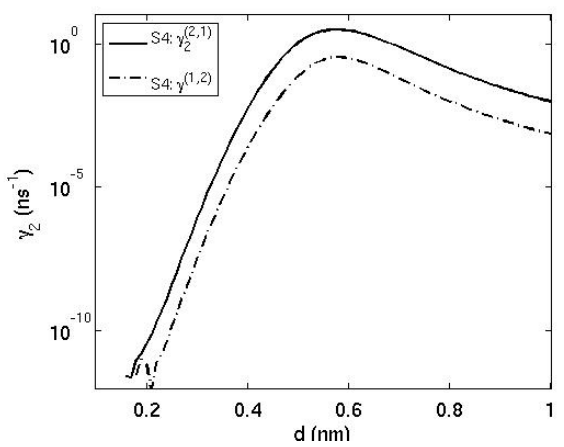

(d)

FIG. 5.3. Fixing $U=-0.05 \mathrm{~V} \mathrm{~nm}^{-1}$, we numerically solve 3.2 and 3.7) and obtain the mean escape times and left splitting probabilities at two states $S_{2}^{(1)}=(-0.9,0)$ and $S_{2}^{(2)}=(0,0.9)$ for d between $0.1 \mathrm{~nm}$ and $1 \mathrm{~nm}$, which determine the escaping rates and transition rates by 3.12). We compare it with the results of the three-state simplified model 5.1. (a) The mean escape time $\tau_{2}$ vs. $d$ at different states. (b) The splitting probability $\rho_{2} v s . d$ at different states. (c) The escaping rates $\beta^{(1)}=\beta_{2}^{-(1,1)}$ and $\beta^{(2)}=\beta_{2}^{+(1,2)}$. (d) The transition rates $\gamma^{(1)}=\gamma_{2}^{(2,1)}$ and $\gamma^{(2)}=\gamma_{2}^{(1,2)}$. Note that those rates only depend on the mean escape time and left splitting probability, and are independent of entry rates.

(black dash-dotted curve) in four-state formulation. For large $d$, the external potential has a large gradient near both ends of the channel (as shown in Fig. 5.1(a)], which pulls the new ion introduced at either source towards the center of channel, and into balance with the existing ion at around $(-0.45,0.45)$. So new entering ions at each source are less likely to leave from the same end of the channel, and both the left splitting probability at left state (black solid curve) and the right splitting probability at right state (black dash-dotted curve) decrease monotonically as $d$ increases. In addition, the left-splitting probability $\rho_{2}$ at the balanced state $S_{2}^{(1)}=(-0.45,0.45)$ in the three-state formulation is plotted by the red solid curve, which overlaps with $\rho_{2}(-0.9,0)$. As with the escape time, the splitting probability is insenstive to the initial position of the ions, and is dominated by the effects of the potential.

Next we compare the escape rates $\beta$ in the two formulations in Fig. 5.3(c) The black solid curve shows the rate at which an ion at left state $S_{2}^{(1)}=(-0.9,0)$ escapes from the left side, the black dash-dotted curve plots the rate at which an ion at right state $S_{2}^{(2)}=$ $(0,0.9)$ escapes from right side. The left and right escaping rates at the balanced state 
$S_{2}^{(1)}=(-0.45,0.45)$ in three-state formulation 5.1 are plotted by red solid curve and red dash-dotted curve, respectively. All escaping rates drop rapidly as $d$ increases. When $d \geq 0.7$, the potential well is so broad that the two ions are trapped in the channel for a long time. In Fig. 5.3(d), we plot the transition rates between states $S_{2}^{(1)}=(-0.9,0)$ and $S_{2}^{(2)}=(0,0.9)$. Due to the inclined voltage, the transition from left state $S_{2}^{(1)}$ to $S_{2}^{(2)}$ occurs more often than the other way, namely the transition rate $\gamma_{2}^{(2,1)}$ depicted by solid curve is above $\gamma_{2}^{(1,2)}$ by the dash-dotted curve. When $d<0.4$, the transition rates are very low $\left(<10^{-4} \mathrm{~ns}^{-1}\right)$, so the two states are very distinct. For large $d$, the observed single state in Fig. 5.2 can be treated as average of the two distinct states.

We remark that the mean escape time of the ion from a one-ion channel $\tau_{1}\left(S_{1}^{(1)}=\{0\}\right)$ is $O\left(10^{5}\right)$ times larger than $\tau_{2}\left(S_{2}^{(1)}\right)$, so the escape rates $\beta_{1}^{-(1,1)} \ll \beta_{2}^{-(1,1)}$ and $\beta_{1}^{+(1,1)} \ll \beta_{2}^{+(1,2)}$. By (3.13) we see that for there to be an appreciable probability of having no ion in the channel we need $\tau_{1}\left(S_{1}^{(1)}\right)^{-1} \sim \alpha_{0}^{-(1,1)}+\alpha_{0}^{+(1,1)}=\lambda+\mu$. In our example, we choose the smallest entry rates to be $\lambda=\mu=1$, so the resulting $\widetilde{P}_{0}^{(1)}$ is extremely small compared to $\widetilde{P}_{1}^{(1)}$ and $\widetilde{P}_{0}^{(1)}$, and is therefore negligible. Thus, for any entry rates $\lambda=O(1), \mu=O(1)$, only one-ion occupancy (small entry rates) or two-ion occupancy (large entry rates) is observed most of time (i.e. $\widetilde{P}_{1}^{(1)}+\widetilde{P}_{2}^{(1)}+\widetilde{P}_{2}^{(2)} \approx 1$ ).

In Fig. 5.4(a) fixing $\lambda=\mu=5 \mathrm{~ns}^{-1}, U=-0.05 \mathrm{~V} \mathrm{~nm}^{-1}$, we compare the stationary probabilities of two-ion occupancy (solid curves) and one-ion occupancy (dash-dotted curve) for various values of $d$ using (i) the four-state formulation (3.8) (black curves), (ii) the threestate formulation (5.1) (red curves), and (iii) by solving the Fokker-Planck equations using Comsol (discrete markers). When $d$ is large, the stationary probabilities obtained from all three methods agree with each other, which confirms that the single balanced state in the three-state formulation can be treated as an average of the two distinct states (with frequent transitions) in the four-state formulation. Because the mean escape time grows from $O\left(10^{-2}\right)$ ns to $O\left(10^{2}\right)$ ns with $d$ increasing as shown in Fig. 5.3(a), a constant entry rate $\lambda=\mu=5$ $\mathrm{ns}^{-1}$ leads to a transition from initially one-ion dominant to two-ion dominant channel.

After obtaining the rates and probabilities, we can compare the flux through the channel from the rate theory and the solution of the Fokker-Planck equations. We integrate the right hand side of $(2.8 \mathrm{~b})$ with respect to $x_{1}$ over $[-L, L]$, which yields

$$
f_{1 R}-f_{1 L}+(\mu+\lambda) I_{0}-\left(\mu \int_{-L}^{x_{+}} \widetilde{P}_{1} d x_{1}+\lambda \int_{x_{-}}^{L} \widetilde{P}_{1} d x_{1}\right)-\left(f_{2 R}-f_{2 L}\right)=0,
$$

where

$$
\begin{aligned}
& f_{2 L}=\int_{-L}^{L} D\left(\frac{\partial \widetilde{P}_{2}}{\partial x_{1}}+\widetilde{P}_{2} \frac{\partial \Phi_{2}}{\partial x_{1}}\right)\left(-L, x_{1}\right) d x_{1}=\int_{-L}^{L} D \frac{\partial \widetilde{P}_{2}}{\partial x_{1}}\left(-L, x_{1}\right) d x_{1}, \\
& f_{2 R}=\int_{-L}^{L} D\left(\frac{\partial \widetilde{P}_{2}}{\partial x_{2}}+\widetilde{P}_{2} \frac{\partial \Phi_{2}}{\partial x_{2}}\right)\left(x_{1}, L\right) d x_{1}=\int_{-L}^{L} D \frac{\partial \widetilde{P}_{2}}{\partial x_{2}}\left(x_{1}, L\right) d x_{1}, \\
& f_{1 L}=D\left(\frac{d \widetilde{P}_{1}}{d x_{1}}+\widetilde{P}_{1} \frac{d \Phi_{1}}{d x_{1}}\right)(-L)=D \frac{d \widetilde{P}_{1}}{d x_{1}}(-L), \\
& f_{1 R}=D\left(\frac{d \widetilde{P}_{1}}{d x_{1}}+\widetilde{P}_{1} \frac{d \Phi_{1}}{d x_{1}}\right)(L)=D \frac{d \widetilde{P}_{1}}{d x_{1}}(L) .
\end{aligned}
$$

In Table 5.1. we show that the eight transitions connected to state $S_{1}^{(1)}$ in Fig. 3.1 correspond one by one to the eight terms in 5.2 . 


\begin{tabular}{|c|c|c|}
\hline Transitions by escaping & Flux from rate theory & Flux from Fokker-Planck \\
\hline$S_{2}^{(1)} \stackrel{\beta_{2}^{-(1,1)}}{\longrightarrow} S_{1}^{(1)}$ & $\beta_{2}^{-(1,1)} \widetilde{P}_{2}^{(1)}$ & $f_{2 L}$ \\
$S_{2}^{(2)} \stackrel{\beta_{2}^{+(1,2)}}{\longrightarrow} S_{1}^{(1)}$ & $\beta_{2}^{+(1,2)} \widetilde{P}_{2}^{(2)}$ & $-f_{2 R}$ \\
$S_{1}^{(1)} \stackrel{\beta_{1}^{+(1,1)}}{\longrightarrow} S_{0}^{(1)}$ & $\beta_{1}^{+(1,1)} \widetilde{P}_{1}^{(1)}$ & $f_{1 L}$ \\
$S_{1}^{(1)} \stackrel{\beta_{1}^{-(1,1)}}{\longrightarrow} S_{0}^{(1)}$ & $\beta_{2}^{-(1,1)} \widetilde{P}_{1}^{(1)}$ & $-f_{1 R}$ \\
$S_{1}^{(1)} \stackrel{\alpha_{1}^{-(1,1)}}{\longrightarrow} S_{2}^{(1)}$ & $\mu \widetilde{P}_{1}^{(1)}$ & $\mu I_{1}$ \\
$S_{1}^{(1)} \stackrel{\alpha_{1}^{+(2,1)}}{\longrightarrow} S_{2}^{(2)}$ & $\lambda \widetilde{P}_{1}^{(1)}$ & $\lambda I_{1}$ \\
$S_{0}^{(1)} \stackrel{\alpha_{0}^{-(1,1)}}{\longrightarrow} S_{1}^{(1)}$ & $\mu \widetilde{P}_{0}^{(1)}$ & $\mu I_{0}$ \\
$S_{0}^{(1)} \stackrel{\alpha_{0}^{+(1,1)}}{\longrightarrow} S_{1}^{(1)}$ & $\lambda \widetilde{P}_{0}^{(1)}$ & $\lambda I_{0}$ \\
\hline
\end{tabular}

Transitions computer by the four-state model 3.8) and the hierarchical Fokker-Planck equation.

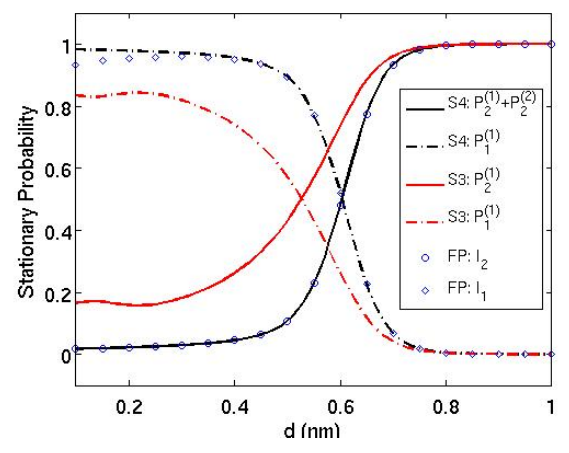

(a)

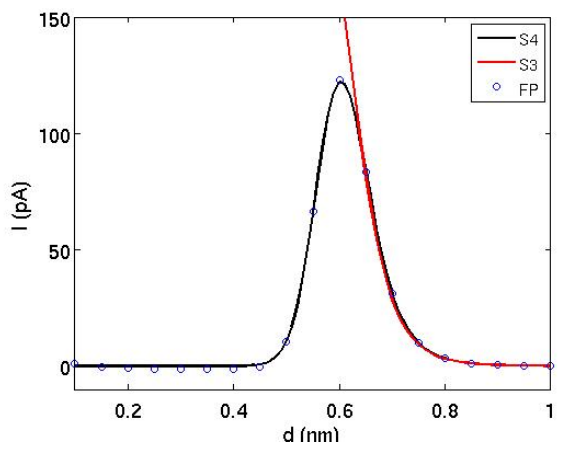

(b)

FIG. 5.4. Fixing $\lambda=\mu=5 \mathrm{~ns}^{-1}, U=-0.05 \mathrm{~V} \mathrm{~nm}^{-1}$. (a) We plot the stationary probabilities of 2-ion and 1-ion by four-state formulation (3.8) (black curves), three-state formulation 5.1) (red curves) and by solving FokkerPlanck equation with 28800 elements (discrete marker). (b) We plot the current I vs. $d$ by four-state formulation 3.8 (black solid curve) and by three-state formulation 5.1 (red solid curve), which are compared with $\left(f_{L}+f_{R}\right)$ in 5.3 from solution of the Fokker-Planck equation (discrete marker).

The left to right flux across the left boundary of the channel is generated by introducing new ions $\mu I_{0}+\mu \int_{-L}^{x_{+}} \widetilde{P}_{1} d x_{1}$, and the right to left flux across the left boundary of the channel is generated by ions leaving the left boundary $f_{1 L}+f_{2 L}$. Similarly we have the left to right flux across the right boundary of the channel is generated by ions leaving the right boundary $-f_{1 R}-f_{2 R}$, and the right to left flux across the right boundary of the channel is generated by introducing new ions $\lambda I_{0}+\lambda \int_{x_{-}}^{L} \widetilde{P}_{1} d x_{1}$. Thus the overall fluxes are

$$
f_{L}=\mu I_{0}+\mu \int_{-L}^{x_{+}} \widetilde{P}_{1} d x_{1}-f_{1 L}-f_{2 L}, \quad f_{R}=-f_{1 R}-f_{2 R}-\lambda I_{0}-\lambda \int_{x_{-}}^{L} \widetilde{P}_{1} d x_{1} .
$$

In Fig. 5.4(b), we fix $\lambda=\mu=5 \mathrm{~ns}^{-1}, U=-0.05 \mathrm{~V} \mathrm{~nm}^{-1}$, and plot the current $I$ vs. $d$ from the flux $\left(f_{L}+f_{R}\right)$ in 5.3 by discrete open diamands, obtained by solving (2.8). In 
comparison, the black solid curve depicts the current obtained by applying the four-state rate theory [3.8, and the red solid curve depicts the current by the three-state rate theory [5.1]. We see that for a broad potential well with $0.6<d<1$, all three methods reach a good agreement: the four-state works for large $d$, even though the stationary probability distribution in Fig. 5.4(a) shows there should be three states for large $d$. This is because of our use of escape times and splitting probabilities to determine the transition rates in the model, rather than by trying to estimate hopping rates directly. However, with small $d$, the three-state model is not an accurate description of the Markov process, and the flux quickly becomes inaccurate.

An important observation from Fig. 5.4(b) is that a maximal current is achieved around $d=0.6 \mathrm{~nm}$, which means there exists an optimal shape of the potential well to conduct ions, even if the depth of the well remains the same. This result agrees with the argument in [1] for a single ion channel with piecewise linear potential energy.

We may explain the existence of an optimal flux by looking at the stationary probabilities $\widetilde{P}_{2}$ and $\widetilde{P}_{1}$ as a function of $d$ in Fig. 5.4(a) When $d$ is small, the potential well is very narrow and steep near the binding site, but relatively flat near the end of channel, so any new ion introduced would escape very quickly from the same end by diffusion, leaving the old ion in the channel. For example, at $d=0.1 \mathrm{~nm}$, both escaping rates $\beta_{2}\left(S_{2}^{-(1,1)}\right)$ and $\beta_{2}\left(S_{2}^{+(1,2)}\right)$ are over $500 \mathrm{~ns}^{-1}$. Thus the channel has only one ion $\left(\widetilde{P}_{1}>0.8\right)$ most of the time, obviously the process of ion entering and leaving from the same end does not generate any through flux. On the other hand, when $d$ is large, the potential well is very broad and flat near the binding site, two ions can hardly escape from the channel, as shown by the large mean escape time in Fig. 5.3(a), once a new ion is introduced to the channel, it quickly moves towards the center, and settles into a balanced state in the well with the other ion; thus the 2-ion state dominates $\left(\widetilde{P}_{2}>0.9\right)$. In this case the flux is small because two ions are trapped in the channel for a long time.

When neither 2-ion or 1-ion occupancy dominates in the channel, so that there are adequate transitions between the 2-ion distinct states and frequent escapes from the 2-ion to the 1 -ion state, a large flux is generated. This explains heuristically why an intermediate potential well has an optimal geometry.

Finally we investigate how the entry rates affect the optimal flux for the family of potential wells in Fig. 5.1(a) using the four-state Markov chain formulation 3.8. Recall that the escape rates $\beta_{2}^{-(1,1)}, \beta_{2}^{+(1,2)}$ and transition rates $\gamma_{2}^{(1,1)}, \gamma_{2}^{(1,2)}$ are determined by the potential through mean escape time and left splitting probability, and thus are independent of the entry rates. We fix the applied field $U=-0.05 \mathrm{~V} \mathrm{~nm}^{-1}$ and plot in Fig. 5.5(a) the stationary probabilities $\widetilde{P}_{2}$ (black) and $\widetilde{P}_{1}$ (red) for $\lambda=1,5,20,100 \mathrm{~ns}^{-1}$. The intersection points of each pair of curves at which $\widetilde{P}_{2}=\widetilde{P}_{1} \approx 0.5$ for $\lambda=1,5,20,100$ are at $d \approx 0.665,0.6,0.54,0.41$ respectively. This illustrates the fact that when the potential well is narrow and steep at the binding site ( $d$ small), the escaping rates $\beta_{2}^{-(1,1)}$ and $\beta_{2}^{+(1,2)}$ are large (shown in Fig. 5.3(c) , so the entry rates have to increase in order to have equal probabilities of 2-ion and 1-ion occupancy.

In Fig. 5.5(b), we plot the current $I(d)$ for $\lambda=1,5,20,100 \mathrm{~ns}^{-1}$. As expected, the current increases as the entry rates increases, but we also find that the value of $d$ at which the current is optimised shifts; the critical values of $d$ at which optimal flux is achieved are respectively $d \approx 0.63,0.6,0.59,0.57$. Thus the optimal value of $d$ slightly decreases as the entry rates increases, which shows that the larger escaping rates of tighter potentials require larger entry rates to optimize the flux.

6. Conclusion. We have presented a set of hierarchical Fokker-Planck equations describing ion permeation in multi-ion channels, and reduced these systematically to a discrete rate theory. The basis of the reduction is the fact that many channels have internal binding 


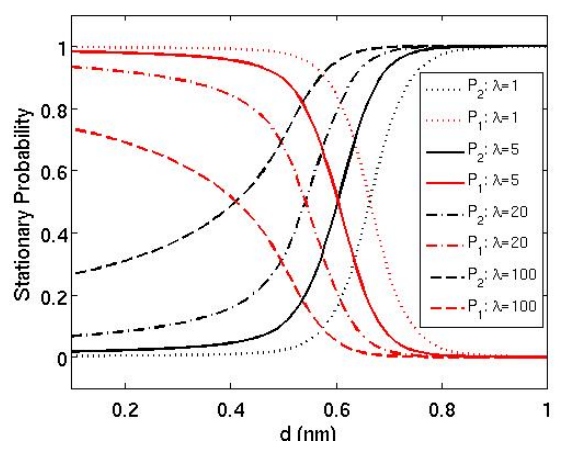

(a)

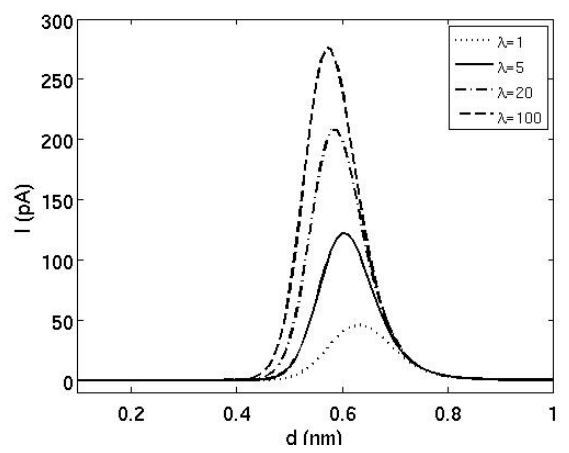

(b)

FIG. 5.5. Fixing $U=-0.05 \mathrm{~V} \mathrm{~nm}^{-1}$. (a) We plot stationary probability $\widetilde{P}_{2}$ (black) and $\widetilde{P}_{1}$ (red) obtained from four-state Markov Chain formulation $\sqrt{3.8}$ for $\lambda=1,5,20,100 \mathrm{~ns}^{-1}$. The intersection points of each pair of curves at which $\widetilde{P}_{2}=\widetilde{P}_{1} \approx 0.5$ for $\lambda=1,5,20,100$ are at $d \approx 0.665,0.6,0.54,0.41 \mathrm{~nm}$, respectively. (b) We plot current obtained from four-state Markov Chain formulation for $\mu=1,5,20,100 \mathrm{~ns}^{-1}$. The critical values of $d$ at which optimal flux is achieved are respectively $d \approx 0.63,0.6,0.59,0.57 \mathrm{~nm}$.

sites at which ions sit, so that ions transport by hopping between sites on a slow time scale while oscillating in the binding sites on a fast time scale. Since the fast oscillation is not key in determining the conduction rate, we can reduce the continuous dynamics to the slow transition between the discrete states, and thus provide an efficient way to calculate the current through the channel. A key component of our reduction was the use of exit times and splitting probabilities to determine the discrete hopping rates, rather than trying to estimate these directly using Kramer's theory for example. This means that the predictions of the discrete model are accurate even when the internal states are not so well defined.

In contrast to traditional Eyring rate theory [11] and the recent study of a one-ion channel in [1], we have developed a general theory for multi-ion channels, and have shown an intricate coupling between transition rates, mean escape time and splitting probability, due to the complexity of the resulting system of Markovian states. The theory is illustrated by a two-ion channel, which is the most accessible example that includes the multi-ion complexity. We have investigated how conductivity of the channel depends on the diffusion coefficient, potential energy landscape, and the ion entry rate. By varying the geometry of the external potential while keeping the depth fixed, we observed that when the potential well is narrow and steep at the binding site, the 1-ion state dominates, but when it is not the 2-ion state dominates. In between there is an optimal geometry which maximizes the ion flux by negotiating between these two extremes and allowing frequent transitions between the 1-ion and 2-ion states.

Acknowledgements. This publication was based on work supported by Award No KUKC1-013-04, made by King Abdullah University of Science and Technology (KAUST). The research leading to these results has received funding from the European Research Council under the European Community's Seventh Framework Programme (FP7/2007-2013)/ ERC grant agreement No. 239870. Radek Erban would also like to thank Somerville College, University of Oxford, for a Fulford Junior Research Fellowship; Brasenose College, University of Oxford, for a Nicholas Kurti Junior Fellowship; the Royal Society for a University Research Fellowship; and the Leverhulme Trust for a Philip Leverhulme Prize. 


\section{REFERENCES}

[1] E. ABAD, J. REINGRUBER, AND M. SANSOM, On a novel rate theory for transport in narrow ion channels and its application to the study of flux optimization via geometric effects, Journal of Chemical Physics, 130 (2009), p. 085101.

[2] B. Alberts, A. Johnson, J. Lewis, M. Raff, K. Roberts, And P. Walter, Molecular Biology of the Cell, Garland Science, New York, 2007.

[3] S. BERNECHE AND B. RouX, Energetics of ion conduction through the $K^{+}$channel, Nature, 414 (2001), pp. 73-77.

[4] - A microscopic view of ion conduction through the $K^{+}$channel, Proceedings of the National Academy of Sciences USA, 100 (2003), pp. 8644-8648.

[5] D. Chen, J. Lear, And B. Eisenberg, Permeation through an open channel: Poisson-Nernst-Planck theory of a synthetic ionic channel, Biophysical Journal, 72 (1997), pp. 97-116.

[6] M. Cheng, A. Mamonov, W. Dukes, and R. Coalson, Modeling the fast gating mechanism in the CIC-O chloride channel, Journal of Physical Chemistry B, 111 (2007), pp. 5956-5965.

[7] K. COOPER, P. GATES, AND R. EISENBERG, Diffusion theory and discrete rate constants in ion permeation, Journal of Membrane Biology, 106 (1988), pp. 95-105.

[8] K. COOPER, E. JAKOBSSON, AND P. WOLYNES, The theory of ion transport through membrane channels, Progress in Biophysics and Molecular Biology, 46 (1985), pp. 51-96.

[9] B. CORRY, S. KUYUCAK, AND S. CHUNG, Test of continuum theories as models of ion channels. II. PoissonNernst-Planck theory versus Brownian dynamics, Biophysical Journal, 78 (2000), pp. 2364-2381.

[10] D. Doyle, J. Morais Cabral, R. A. Pfuetzner, A. Kuo, J. Gulbis, S. Cohen, B. Chait, And R. MACKINNON, The structure of the potassium channel: molecular basis of $\mathrm{K}^{+}$conduction and selectivity, Science, 280 (1998), pp. 69-77.

[11] H. EYRING, The activated complex and the absolute rate of chemical reactions, Chemical Reviews, 17 (1935), pp. 65-77.

[12] M.B. FlegG, S.J. CHAPMAN, AND R. ERBAN, The two-regime method for optimizing stochastic reactiondiffusion simulations, Journal of the Royal Society Interface, 9 (2012), pp. 859-868.

[13] B. Franz, M.B. FlegG, S.J. ChapMAn, AND R. ERBAN, Multiscale reaction-diffusion algorithms: PDEassisted Brownian dynamics. 23 pages, available as http://arxiv.org/abs/1206.5860 2012.

[14] M. Jensen, D. Borhani, K. Lindorff-Larsen, P. MaragaKis, V. Jogini, M. Eastwood, R. DROR, AND D. SHAW, Principles of conduction and hydrophobic gating in $K^{+}$channels, Proceedings of the National Academy of Sciences USA, 107 (2010), pp. 5833-5838.

[15] D. LEVITT, Modelling of ion channels, Journal of General Physiology, 113 (1999), pp. 789-794.

[16] G. Moy, B. Corry, S. KuYUCAK, AND S. Chung, Tests of continuum theories as models of ion channels. I. Poisson-Boltzmann theory versus Brownian dynamics, Biophysical Journal, 78 (2000), pp. 2349-2363.

[17] B. NADler, Z. Schuss, A. Singer, AND R. EISENBERG, Ionic diffusion through confined geometries: from Langevin equations to partial differential equations, Journal of Physics: Condensed Matter, 16 (2004), pp. S2153-S2165.

[18] P. Pongprayoon, O. Beckstein, C. Wee, And M. Sansom, Simulations of anion transport through OprP reveal the molecular basis for high affinity and selectivity for phosphate, Proceedings of the National Academy of Sciences USA, 106 (2009), pp. 21614-21618.

[19] J. PROCOPIO AND O. ANDERSEN, Ion tracer fluxes through gramicidin A modified lipid bilayers, Biophysical Journal, 25(2 Pt 2) (1979), p. 8 a.

[20] S. Redner, A Guide to First-Passage Processes, Cambridge University Press, 2001.

[21] Z. Schuss, B. NADler, AND R. EISEnBERG, Derivation of Poisson and Nernst-Planck equations in a bath and channel from a molecular model, Physical Review E, 64 (2001), p. 036116.

[22] W. VAn Gunsteren And H. Berendsen, Algorithms for Brownian dynamics, Molecular Physics, 45 (1982), pp. 637-647. 